\title{
A Method to Quantify Molecular Diffusion within Thin Solvated Polymer Films: A Case Study on Films of Natively Unfolded Nucleoporins
}

\author{
Rickard Frost, Delphine Débarre, Saikat Jana, Fouzia Bano, Jürgen Schünemann, Dirk Görlich, \\ and Ralf P. Richter*
}

Cite This: ACS Nano 2020, 14, 9938-9952

Read Online

ACCESS | Lلll Metrics \& More | 回 Article Recommendations | S1 Supporting Information

ABSTRACT: We present a method to probe molecular and nanoparticle diffusion within thin, solvated polymer coatings. The device exploits the confinement with well-defined geometry that forms at the interface between a planar and a hemispherical surface (of which at least one is coated with polymers) in close contact and uses this confinement to analyze diffusion processes without interference of exchange with and diffusion in the bulk solution. With this method, which we call plane-sphere confinement microscopy (PSCM), information regarding the partitioning of molecules between the polymer coating and the

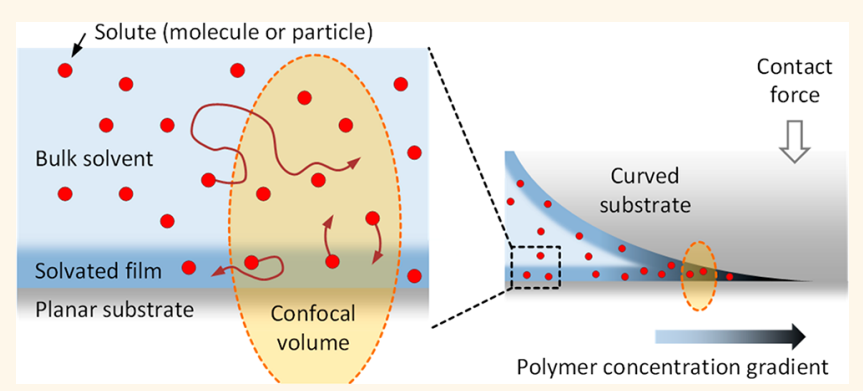
bulk liquid is also obtained. Thanks to the shape of the confined geometry, diffusion and partitioning can be mapped as a function of compression and concentration of the coating in a single experiment. The method is versatile and can be integrated with conventional optical microscopes; thus it should find widespread use in the many application areas exploiting functional polymer coatings. We demonstrate the use of PSCM using brushes of natively unfolded nucleoporin domains rich in phenylalanine-glycine repeats (FG domains). A meshwork of FG domains is known to be responsible for the selective transport of nuclear transport receptors (NTRs) and their macromolecular cargos across the nuclear envelope that separates the cytosol and the nucleus of living cells. We find that the selectivity of NTR uptake by FG domain films depends sensitively on FG domain concentration and that the interaction of NTRs with FG domains obstructs NTR movement only moderately. These observations contribute important information to better understand the mechanisms of selective NTR transport.

KEYWORDS: diffusion, absorption, confinement, polymer film, reflection interference contrast microscopy,

fluorescence recovery after photobleaching; permeability barrier

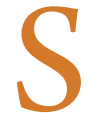

olvated polymer films at the solid-liquid interface constitute a wide span of surface coatings, including polymers physically adsorbed or grafted to/from a supporting surface (planar, structured, or particulate). Such polymer films may be either passive or responsive to external stimuli, e.g., changes in temperature, $\mathrm{pH}$, ionic strength, or light. To physically adsorb polymers onto a solid surface is a simple surface functionalization procedure and can be accomplished using methods such as dip-coating, etc. Similarly, more advanced surface adlayers may be built layer-by-layer through sequential exposure to oppositely charged polyions. Grafting of polymers to/from a solid support requires more specific surface chemistry approaches but generally results in a more durable surface coating. An important subcategory of surface-grafted polymers is polymer brushes. In such surface coatings, polymers are one-end grafted at high density to the solid support, forming a brush-like structure. ${ }^{1,2}$ Independent of the surface functionalization strategy, confinement of polymers in a surface-associated layer affects their conformation and selforganization. Thus, the properties of the polymer are different when associated with a surface compared to when present in

Received: April 6, 2020

Accepted: July 15, 2020

Published: July 15, 2020 


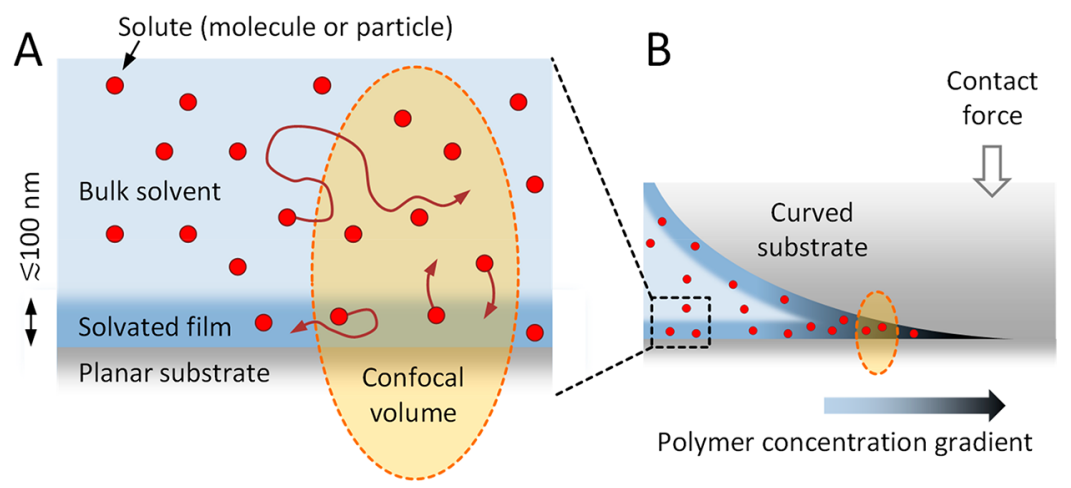

Figure 1. Schematic representation of the problem at hand and the experimental approach. (A) Probe solutes (red dots) partition between the bulk solution and a thin, solvated polymer film. They diffuse in these phases with rates $D_{\text {bulk }}$ and $D_{\text {film }}$, respectively. The objective is to quantify $D_{\text {film }}$. Because the probe molecules continuously move between the two phases and the polymer film thickness is below the optical resolution limit, it is challenging to separate $D_{\text {film }}$ from $D_{\text {bulk }}$ (B) By confining the polymer coating(s) between a planar and a curved surface, the bulk solution is excluded in the region surrounding the contact between the two surfaces. This enables optical microscopy to probe diffusion within the polymer film(s) and also to quantify the partitioning of probe molecules between the bulk and polymer phases. The gradual compression of the polymer film(s) near the contact point also entails a polymer concentration gradient that can be exploited to measure the diffusion and partitioning of probe molecules as a function of polymer film compression and concentration. Note that the contact geometry was stretched along the vertical axis for illustrative purposes: in reality, the gap height increases very slowly with distance from the contact point, and the confocal volume will always include the entire thickness of the gap across the imaged area.

bulk solution. Furthermore, and importantly, the polymer film may significantly alter and enable tuning of the properties of the solid surface.

During the last few decades, solvated polymer coatings have been investigated for a broad range of applications, from fundamental research to everyday-life applications. Examples include reconstituted biomolecular and biomimetic films, ${ }^{3}$ biomaterials, ${ }^{4}$ biosensors, ${ }^{5}$ nanomedicine, antifouling and antimicrobial coatings, ${ }^{6,7}$ purification and separation membranes, food processing, paints, lubrication, 8 and energy storage. ${ }^{9}$ An important functional parameter of such coatings is how the constituent polymers and active substances (e.g., active synthetic molecules, proteins, nanoparticles, viruses; here collectively called solutes) diffuse within them. Depending on the application, one may design ways to either enhance or delay such diffusion. Consequently, there is a broad need for the analysis and quantification of diffusion processes within thin polymer films.

However, this is currently challenging when the film is immersed in a solvent phase. Methods based on optical microscopy, such as fluorescence recovery after photobleaching (FRAP), fluorescence correlation spectroscopy (FCS), and single-particle tracking (SPT), ${ }^{10}$ are well-established and popular to study diffusion processes. FRAP and FCS though fail for thin films that dynamically exchange solutes with the bulk solution: because the dimensions of the volume probed (>200 $\mathrm{nm}$ in $x y$ and $>500 \mathrm{~nm}$ in $z$, for diffraction-limited confocal optics) exceed the film thickness ( $\lesssim 100 \mathrm{~nm})$, diffusion scenarios including (i) diffusion within the film, (ii) diffusion in the adjacent bulk liquid, and (iii) exchange between the film and the bulk all contribute to the optical signal (as illustrated in Figure 1A); in this scenario, it is thus challenging to separate in-film diffusion from the other two processes. Similarly, although single-particle tracking is able to determine diffusion coefficients in smaller spaces, statistical analysis becomes limited when diffusion trajectories within the film are short owing to rapid exchange between the film and the bulk solution.

Here, we present an analytical methodology, based on optical microscopy, that overcomes this limitation by confining polymer film(s) between two surfaces, one planar and the other macroscopically curved (as illustrated in Figure 1B). The confined volume near the contact point retains nanometer dimensions along the optical axis, inferior or comparable to the thickness of the polymer film, yet at the same time the lateral dimensions are micrometric and thus large enough to be resolved with conventional microscopy. The setup effectively excludes the bulk solution from a region close to the surfacesurface contact so that solute diffusion within the film can be probed and confounding solute exchanges with the bulk are excluded. This concept solves an important problem in thin film analysis for which there is currently no solution. Using the same approach, partitioning of solutes between the polymer film and the bulk liquid can also be readily measured. In addition, because the contact force between the surfaces can be set and the geometry of the interface is known, a defined gradient of polymer compression and concentration is created and it becomes possible to measure solute diffusion and partitioning as a function of these parameters in a single measurement. This substantially extends the capability of the methodology.

It should be noted here that the generation of confined spaces using a plane-sphere geometry is a well-known procedure. The surface force apparatus, for example, combines this geometry with exquisite sensitivity in force and separation distance, ${ }^{11}$ to measure interaction forces between functionalized surfaces; more recently, this approach has also been combined with optical analysis for concurrent studies of the molecular organization and diffusion within the confined fluids by microscopy and spectroscopy techniques. ${ }^{12-15}$ Plane-sphere geometries have also already been combined with optical microscopy for improved single-molecule imaging ${ }^{16-18}$ or to visualize dynamic processes under confinement as diverse as blood clot formation, ${ }^{19}$ lubricant transfer during interfacial shear, ${ }^{20}$ and capillary condensation. ${ }^{21}$ Distinct aspects of the here-described method are the application to solvated polymer films and its ease of integration with existing microscopes and imaging modalities, where a substantially static and constant contact force is beneficial to control the compression of the polymer film. 
A
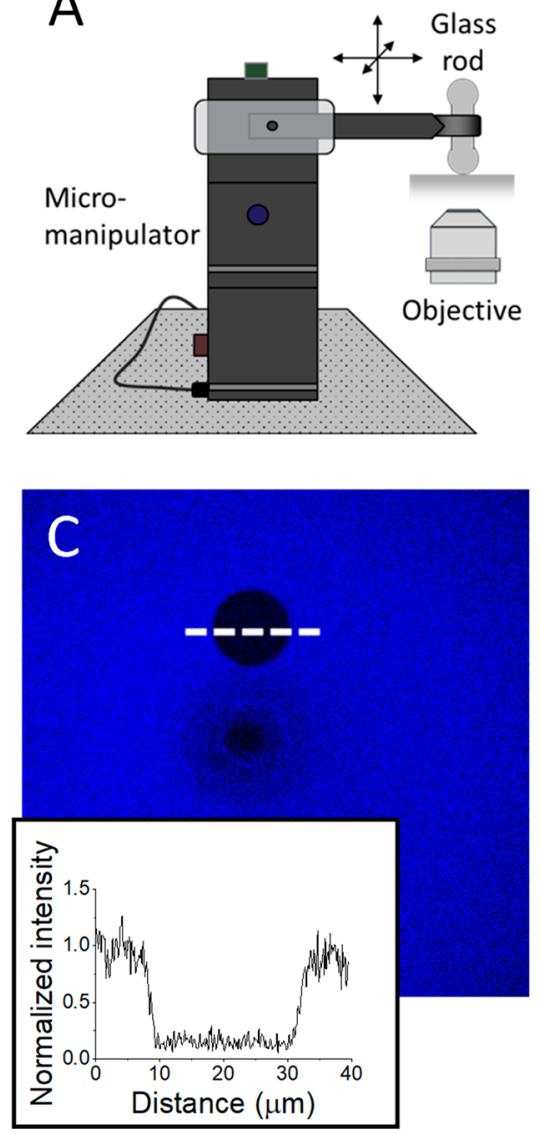
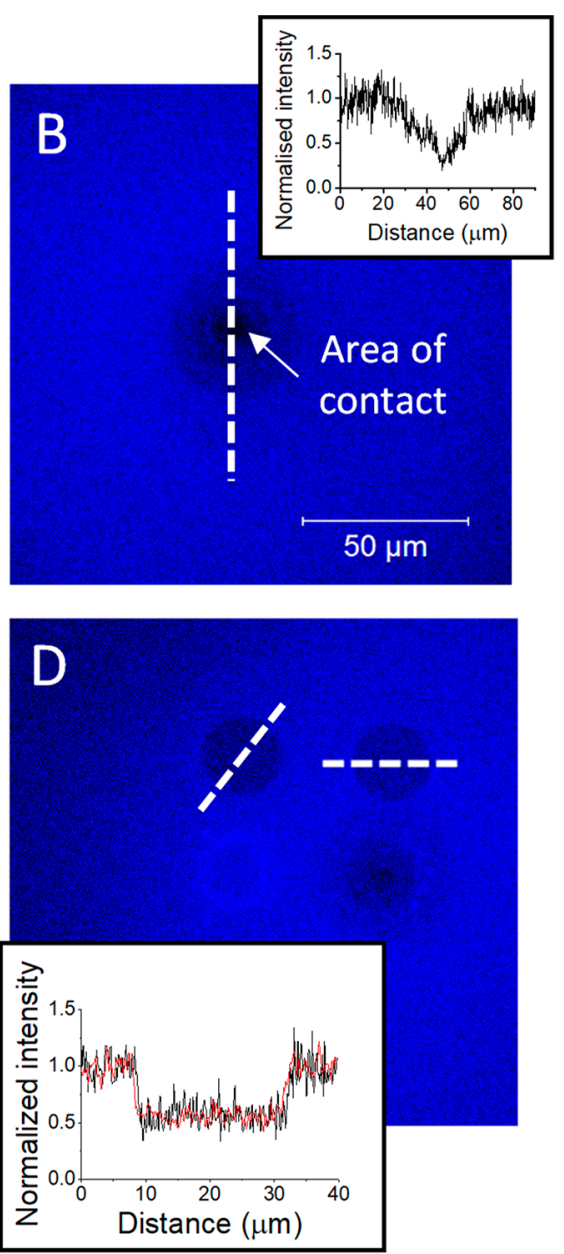

Figure 2. Confinement of polymer films at the plane-sphere interface. (A) Schematic representation of the experimental setup (not to scale). A glass rod with a hemispherical cap is coarse-aligned with the optical axis, and lowered toward a planar surface using a micromanipulator until contact is reached. (B) Fluorescence micrograph of the plane-sphere interface with both surfaces functionalized with $\mathrm{FG}^{\mathrm{Nsp1}}-\mathrm{His}_{10}$ films $\left(1 \mathrm{~mol} \%\right.$ of $\mathrm{FG}^{\mathrm{Nsp1}}-\mathrm{His}_{10}$ was labeled with the fluorophore Atto488). The area of contact is visible as a zone of reduced fluorescence. Inset shows fluorescence intensity profile taken along the white dashed line. (C) Interface shown in (B) after photobleaching of a circular region. The lack of apparent recovery demonstrates that $\mathrm{FG}^{\mathrm{Nsp} 1}$ was bound and immobile on the surfaces. (D) Interface shown in (C) after retracting the spherical surface and making a new contact approximately $50 \mu \mathrm{m}$ to the right. Comparison of fluorescence intensity profiles (insets in (C) and (D), taken along the white dashed lines in (C) and (D)) shows both surfaces were functionalized with $\mathrm{FG}^{\mathrm{Nsp1}}-\mathrm{His}_{10}$ at comparable densities.

The methodology, which we call plane-sphere confinement microscopy (PSCM), uses reflection interference contrast microscopy (RICM), to analyze the gap profile between the apposed surfaces (which is defined by the shape of the surfaces, the applied load, and the thickness and compressibility of the polymer films); fluorescence microscopy, to image the distribution of probe molecules around the contact point; and FRAP, to probe diffusion of probe molecules within the confined polymer films. We demonstrate the use of PSCM using model systems of the nuclear pore permselectivity barrier, an important biological confined polymer matrix that makes the transport of macromolecules between the cell nucleus and the cytoplasm highly selective. ${ }^{22}$

\section{CASE STUDY: THE NUCLEAR PORE PERMSELECTIVITY BARRIER}

The presented analytical methodology, PSCM, is generic and applicable to a wide range of polymer film systems. To demonstrate the use of the methodology, we have selected a biomimetic system of the nuclear pore permselectivity barrier.
Nuclear pore complexes (NPCs) control the exchange of biomolecules between the nucleus and the cytoplasm of all eukaryotic cells. ${ }^{22}$ NPCs perforate the nuclear envelope and through selective transport of RNA and proteins enable the spatial separation of transcription (cell nucleus) and translation (cytoplasm), which provides a powerful mechanism to control gene expression. Although small molecules up to roughly $5 \mathrm{~nm}$ in diameter can diffuse freely across the NPC, the passage of larger macromolecules is impeded unless they are bound to nuclear transport receptors (NTRs). ${ }^{23}$ The NPC consists of a scaffold of folded proteins that defines an approximately $40 \mathrm{~nm}$ wide channel. The channel, however, is not empty but filled with a meshwork of specialized natively unfolded protein domains that are rich in phenylalanine-glycine (FG) dipeptides (FG domains), and acts as a selective permeation barrier. $^{24}$ For example, it has been shown that NTRs are substantially enriched in FG domain protein films. ${ }^{25}$ NTRs tend to have many binding sites for FG dipeptide motifs; that is, the interactions between NTRs and FG domains are intrinsically multivalent. Recent studies have found that the 
thermodynamic and morphological aspects of NTR binding to FG domain assemblies can be described well by simple models that consider the FG domains as homogeneous flexible polymers and the NTRs as featureless spheres. This indicates that detailed structural features of FG domains and NTRs are secondary to function and that simple soft matter physics models are able to capture essential features of the system. ${ }^{25,26}$ Each FG domain typically contains tens of FG motifs, which contribute to intra- and intermolecular interactions of FG domains, as well as to the binding of NTRs. The attractive interactions between FG domains are essential for the functionality of the permeability barrier. ${ }^{27}$ Permselectivity consists of three basic, sequential steps: (I) entry into the pore, (II) diffusion through the pore, and (III) release from the pore. While steps (I) and (III) have been studied in detail and begun to be understood, much less is known about step (II). Studies using intact nuclear pores have shown that translocation can occur fast (within milliseconds). ${ }^{28-30}$ Detailed analysis of single-molecule tracks by Yang and Musser ${ }^{29}$ revealed diffusion of a selected NTR cargo when interacting with the nuclear pore complex is only moderately (i.e., less than 10-fold) reduced compared to diffusion in the cytoplasm. Although it has been revealed that individual NTR-FG motif interactions are extremely fast, ${ }^{31}$ it still remains to be determined how diffusion through the channel can occur rapidly with respect to collective NTR-FG motif interactions (i.e., the multivalent interactions between a given NTR and the FG motifs presented by the surrounding meshwork of FG domains). A main impediment within this area of research has been that techniques are lacking to study the diffusion process within confined spaces such as the NPC or other nanoscale phases.

\section{A RECONSTITUTED MODEL OF THE NUCLEAR PORE PERMSELECTIVITY BARRIER}

Films of end-grafted FG domains (such as FG domain brushes $)^{3,32,33}$ have been successfully used as a model system to study the properties and mechanisms of function of the nuclear pore permselectivity barrier. Past work using this model system mainly focused on morphology ${ }^{3,32,34}$ (e.g., film thickness changes and phase formation) and thermodynamic parameters $^{3,25,35,36}$ (e.g., partitioning of NTRs between the bulk phase and the FG domain film). Here, we use PSCM to extract information regarding both partitioning and diffusion of probe molecules within FG domain films. As probe molecules we utilize enhanced green fluorescent protein $\left(\mathrm{GFP}^{\mathrm{Std}}\right)$, a GFP mutant designed not to bind to FG domains (GFP ${ }^{\text {Inert }}$ ), and a GFP mutant designed to gain NTR-like properties $\left(\mathrm{GFP}^{\mathrm{NTR}}\right){ }^{37}$ We thus demonstrate the use of PSCM and quantify the diffusion coefficient of an NTR-like protein within an ultrathin film of FG domains.

\section{RESULTS AND DISCUSSION}

We introduce plane-sphere confinement microscopy with the purpose to allow studies of diffusion processes within solvated polymer films at the solid-liquid interface. A planar and a semispherical surface, both functionalized with the polymer film of interest, were brought into contact in a well-controlled fashion using a micromanipulator (Figure 2A). Thus, close to the point of contact between the planar and spherical surface the polymer films will overlap, excluding all bulk liquid. This is the region of primary interest for PSCM: thanks to the large size of the hemisphere, its lateral dimensions will exceed $10 \mu \mathrm{m}$ for polymer coatings of $>10 \mathrm{~nm}$ in thickness (vide infra). Processes on this length scale can be readily resolved by fluorescence microscopy, thus enabling the characterization of diffusion processes inside the polymer film without interference from the bulk solution. We will first demonstrate how the confined geometry is realized and characterized and then describe how information regarding the diffusion coefficient of the fluorescent probe molecule (solute) and its partitioning between the polymer film and the bulk solution can be extracted.

Defining the Confined Space between PolymerCoated Plane and Sphere. Polymer Coatings. To anchor FG domains of Nsp1 ( $\left.\mathrm{FG}^{\mathrm{Nsp} 1}\right)$ to desired surfaces, we exploited the specific binding of poly histidine tags (located at the $\mathrm{C}$-terminus of $\mathrm{FG}^{\mathrm{Nsp} 1}$ ) to $\mathrm{Ni}^{2+}$-EDTA moieties on the two surfaces. The process to prepare films of C-terminally grafted $\mathrm{FG}^{\mathrm{Nsp} 1}$ in this way has previously been established ${ }^{33}$ and was here validated by quartz crystal microbalance (QCMD) on an identically functionalized reference sensor surface (Supporting Figure S1). In previous work, we also demonstrated how the surface density of FG domain films can be quantified by spectroscopic ellipsometry (SE) and that QCM$\mathrm{D}$ and SE data can be correlated to estimate surface densities from the QCM-D response., ${ }^{3,33,25}$ Building on this prior work, we estimate that the $\mathrm{FG}^{\mathrm{Nsp} 1}$ film used here has a surface density of $5 \pm 1 \mathrm{pmol} / \mathrm{cm}^{2}$ (equivalent to a root-mean-square anchor distance of $5.9 \pm 0.6 \mathrm{~nm}$; see Supporting Figure S1 for details). Moreover, extensive analysis by atomic force microscopy nanoindentation, QCM-D, and SE had previously revealed the thickness of films of C-terminally anchored $\mathrm{FG}^{\mathrm{Nsp} 1}$ at around $5 \mathrm{pmol} / \mathrm{cm}^{2}$ to be $d_{\mathrm{FG}} \approx 30 \mathrm{~nm}{ }^{34}$ Thus, the uncompressed FG domain film has a mass concentration of $107 \mathrm{mg} / \mathrm{mL}$ and harbors a total molar concentration of $55 \mathrm{mM}$ FG dipeptides (each Nsp1 FG domain features 33 FG dipeptides ${ }^{25}$ ).

The method of FG domain surface grafting was then transferred to planar glass coverslips and rods with a hemispherical cap. Both types of surfaces were functionalized in situ and kept in working buffer at all times during and after FG domain film formation. Aided by a micromanipulator, the rod with a hemispherical cap was aligned with the microscope objective and then lowered toward the planar surface until contact was reached (Figure 2A). The alignment procedure allowed the contact point between the two surfaces to be positioned in the center of the field of view upon first contact (Supporting Figure S2).

To confirm successful FG domain film formation in the PSCM setup, we incorporated $1 \mathrm{~mol} \%$ of $\mathrm{FG}^{\mathrm{Nsp} 1}$ labeled with Atto488 at the free $\mathrm{N}$-terminus and visualized the surface coatings in plane-sphere confinement geometry using confocal microscopy (Figure 2B). The fluorescence micrograph did not show any appreciable features (except in and close to the contact area, vide infra) as expected for homogeneous FG domain films. To probe how the FG domain films on the two apposed surfaces compare, a circular area close to the contact point was first photobleached, and the rod with hemispherical cap was then withdrawn, translated to the right by approximately $50 \mu \mathrm{m}$, and brought back into contact (Figure $2 \mathrm{C}, \mathrm{D}$ ). This procedure revealed that $\mathrm{FG}^{\mathrm{Nsp} 1}$ was present on both surfaces at comparable surface density because the bleaching effect was split into two equal parts where the total loss of intensity in the two spots $(2 \times 45 \%$; 
A
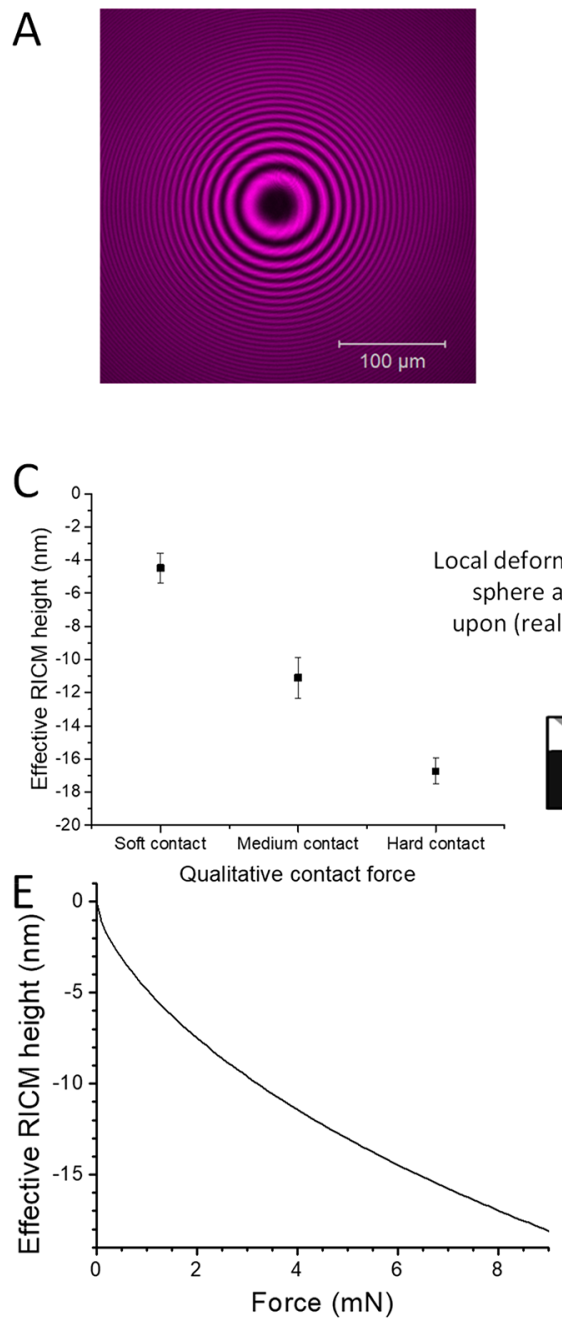

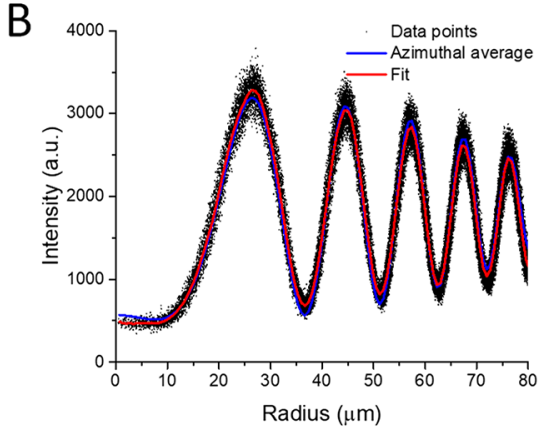

D
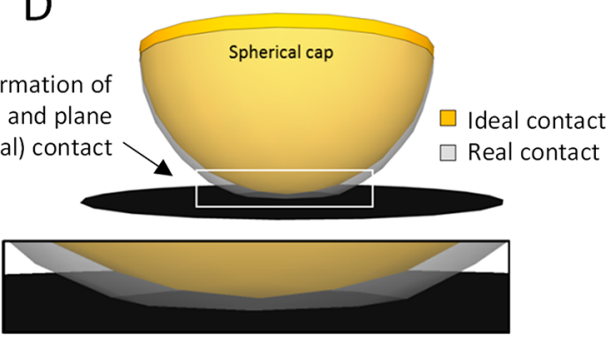

$\mathrm{F}$

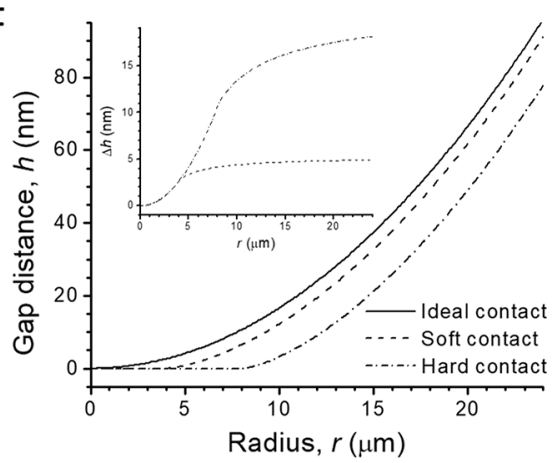

Figure 3. Analysis of gap profiles and contact forces by RICM. (A) Representative RIC micrograph of a spherical surface (glass rod) pressing on a planar surface (coverslip). Conditions: "soft" contact, wavelength of light $\lambda=633 \mathrm{~nm}$. (B) Radial intensity profile extracted from (A) (black dots), azimuthally averaged (blue line) and fitted with an optical model assuming perfect (ideal contact) plane-sphere geometry (red line). (C) Effective RICM height at the center as a function of the quality of the contact, here operationally defined as "soft" (corresponding to a few $\mu \mathrm{m}$ of micromanipulator $z$ motion following initial contact), "medium" $(\sim 8 \mu \mathrm{m})$, and "hard" $(\sim 16 \mu \mathrm{m})$ contact. Data points represent mean \pm standard deviation of five measurements with bare surfaces. (D) Schematic representation of the contact geometry corresponding to an ideal plane-sphere interface (zero contact force; yellow spherical cap) and a real contact (where both surfaces are deformed at the interface owing to the finite contact force; transparent gray spherical cap). (E) Effective RICM height versus compressive force predicted from the Hertz contact model considering the geometries and mechanical properties of the glass rod and the coverslip. The curve is for two bare surfaces; if a polymer film is present between the surfaces, then the effective RICM height can be increased by the optical thickness of the fully compressed polymer film to a good approximation (for details see Supporting Methods, Estimate of compressive forces between sphere and plane). (F) Radial gap profile for "soft" ( $F=1 \mathrm{mN}$; dashed line) and "hard" ( $8 \mathrm{mN}$; dash-dotted line) contact; the idealized case of a perfect plane-sphere contact $(0 \mathrm{mN}$; solid line $)$ is also shown for comparison. The inset shows the difference in gap sizes $(\Delta h)$ between the soft and hard contacts compared to the ideal contact.

inset Figure 2D) was identical to the total loss of intensity in the original spot (90\%; inset Figure 2C).

Moreover, the consistently sharp transition of the fluorescence intensity levels at the periphery of the bleached spot(s) demonstrates that the FG domains are essentially immobile and do not migrate appreciably across the surface within experimentally relevant times. It is notable that the area of contact consistently appeared darker than the surrounding when Atto488-labeled $\mathrm{FG}^{\mathrm{Nsp} 1}$ was used (inset Figure 2B); the fluorescence though largely recovered upon separation of sphere and plane (Figure 2C,D). This suggests that the strong compression in and close to the contact area affected the fluorophore, but the FG domain film remained largely stable during contact. Indeed, a photobleaching assay confirmed the
FG domains remain unable to migrate upon their compression near the contact area (Supporting Figure S3). The exact mechanism for the reduction in fluorescence is not clear. For two FG domain films (each at $5 \mathrm{pmol} / \mathrm{cm}^{2}$ ) with $1 \mathrm{~mol} \%$ Atto488, the projected (onto the interfacial plane) root-meansquare distance of fluorophores is $40 \mathrm{~nm}$. This is much larger than the Förster distance of Atto488 (5 nm), and selfquenching is thus unlikely if the fluorophore is homogeneously distributed in the film. We note though that the FG domains in the very contact zone are strongly compressed and reduced in their hydration (vide infra); this could possibly lead to local microphase separation of the Atto488 fluorophore or reduce the efficiency of the fluorophore in other ways. 

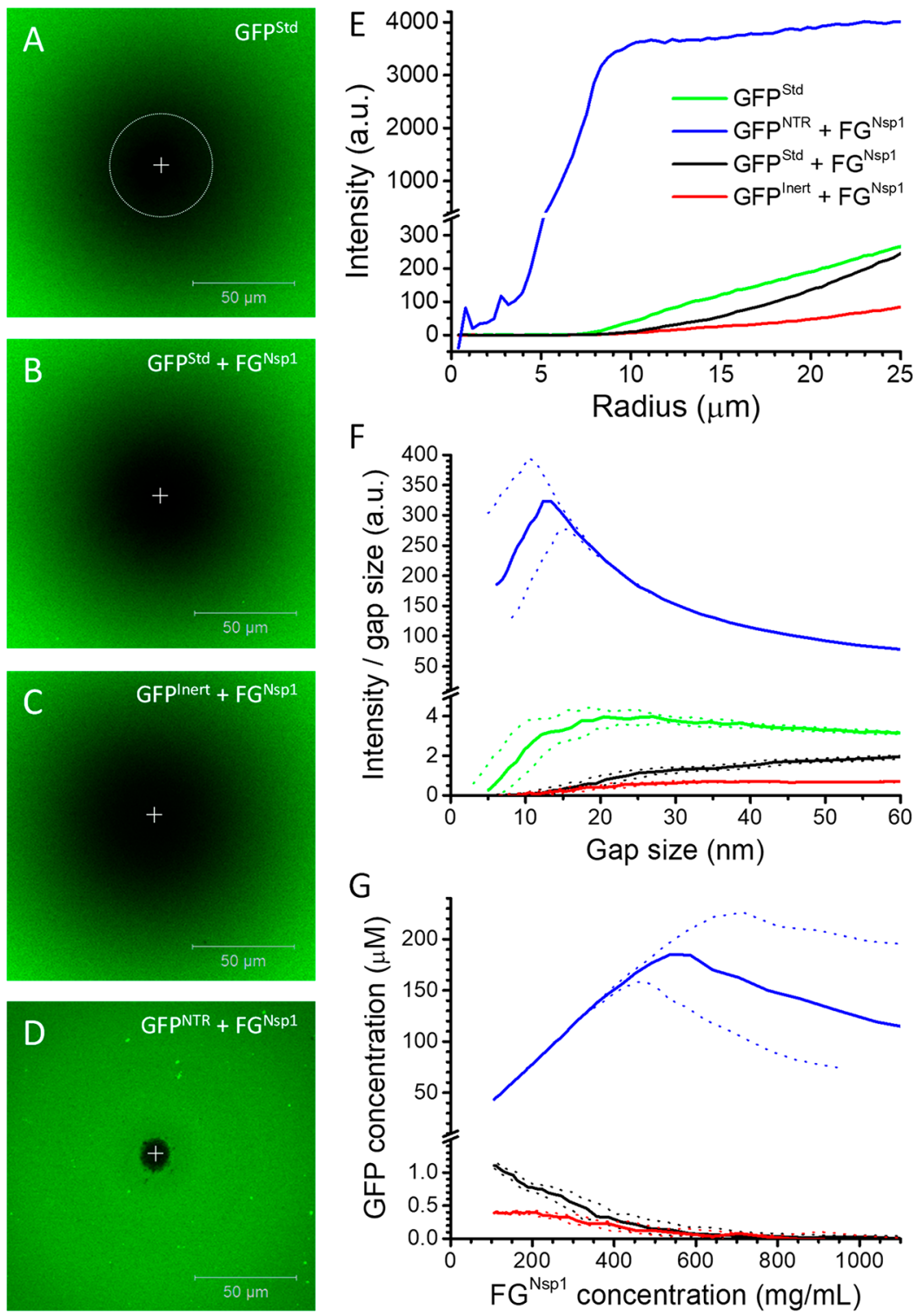

Figure 4. Analysis of GFP distribution inside the polymer film with fluorescence microscopy. (A) Fluorescence micrograph of GFP ${ }^{\text {Std }}$ surrounding the contact area (the white cross indicates the location of the center and the diameter of the contact area) between bare planar and spherical surfaces. (B) As in (A) but with both surfaces functionalized with a FG $^{\text {Nsp1 }}$ film. (C, D) As in (B) but with GFP ${ }^{\text {Inert }}$ (C) and $\mathrm{GFP}^{\text {NTR }}$ (D) instead of $\mathrm{GFP}^{\text {Std }}$. (E) Integrated radial intensity profiles derived from the micrographs in (A)-(D). The white dotted circle in (A) illustrates the area analyzed (the radius was measured from the center of the contact area). (F) Intensity profiles of micrographs in (A) (D), normalized with the gap size between the planar and the spherical surface, plotted versus the gap size (upper curve for each sample). Solid lines were computed with the most probable gap size; dotted lines delineate the confidence interval based on the estimated $\pm 2 \mathrm{~nm}$ uncertainty in gap size. A gap size of $60 \mathrm{~nm}$ here occurs at a radius of approximately $20 \mu \mathrm{m}$. (G) Same as (F) but recalculated to GFP concentration and plotted versus the $\mathrm{FG}^{\mathrm{Nsp1}}$ concentration.

Gap Profile and Contact Force. The interference of light reflected at the plane-solution and solution-sphere interfaces gives rise to a pattern of Newtonian rings. We exploited the capacity of the laser scanning microscope to acquire images of the reflected light, and such reflection interference contrast (RIC) micrographs were then analyzed to quantify gap sizes and, indirectly, the contact force.

A representative RIC micrograph is shown in Figure 3A for a plane-sphere interface without a polymer interlayer. The Newtonian rings appear symmetric and without appreciable imperfections, confirming that both surfaces have the expected smooth finish. The radial intensity profile (Figure 3B) could be fitted with an optical model assuming perfect plane-sphere geometry. However, the effective gap sizes at the center of the contact thus computed were consistently negative and increased in magnitude with the applied force (Figure 3C). This indicated that there were significant deviations from the assumed ideal plane-sphere contact geometry. We hypothesized that these are due to the compressive force entailing the deformation of the planar and spherical surfaces (Figure 3D). To verify this assumption, we computed the shape of the contacting surfaces as a function of compressive force using the Hertz contact model (see Supporting Methods, RICM analysis of a sphere pressing on a planar surface). Subjecting the corresponding idealized theoretical RICM intensity profiles to the above-mentioned optical model indeed generated fits of 
good quality with negative and force-dependent effective gap sizes (Figure 3E), analogous to the experimental data. Moreover, we compared the applied forces predicted for what we operationally defined as "soft", "medium", and "hard" contact in our experiments with rough estimates of the applied forces based on the magnitude of the micromanipulator's $z$ motion and the mechanics of the lever arm to which the glass rod was attached (see Supporting Methods, Estimate of compressive forces between sphere and plane). These were in good agreement, thus demonstrating that the RIC micrographs in conjunction with the Hertz model can be exploited to estimate the contact force and to quantify the real radial gap profile as a function of the distance from the center of contact (i.e., taking into account the deformation of the planar and spherical surfaces upon contact; Figure 3F).

The above-described validation experiments were performed with bare surfaces. RICM, however, can also be used to quantify the contact force and, subsequently, the gap profile in the presence of a polymer interlayer provided that the optical thickness of the compressed interlayer is known (see Supporting Methods, RICM analysis of a sphere pressing on a planar surface). From the RICM analysis, we estimate the compressive forces $F$ in our setup ranged from $1 \mathrm{mN}$ at "soft" contact to $8 \mathrm{mN}$ at "hard" contact (Figure $3 \mathrm{C}$ and E). It can be estimated (by considering the osmotic pressure in the FG domain film; see Supporting Methods, FG domain film thickness under strong compression) that forces of this magnitude would compress the FG domain film to an extent that virtually all solvent is squeezed out, essentially, leaving an incompressible polypeptide melt in the area of contact. From the $\mathrm{FG}^{\mathrm{Nsp} 1}$ grafting density of $5 \mathrm{pmol} / \mathrm{cm}^{2}$, the thickness of the compressed FG domain film would be $2.3 \pm 0.5 \mathrm{~nm}$. Considering also the presence of the EDTA surface functionalization (which is used to graft $\mathrm{FG}^{\mathrm{Nsp} 1}$ via its polyhistidine tag; $\left.{ }^{33} 0.7 \pm 0.2 \mathrm{~nm}\right)$, we can estimate that this compact organic film has a thickness of $d_{\text {compact }}=3.0 \pm 0.7 \mathrm{~nm}$ (ibid.).

All measurements presented in the subsequent sections of the article were performed at soft contact (and without any fluorescently labeled $\mathrm{FG}^{\mathrm{Nsp} 1}$ ). We hence used the appropriate gap profile shown in Figure 3F whenever data for two bare surfaces were analyzed and augmented these values by $2 \times 3$ $\mathrm{nm}=6 \mathrm{~nm}$ when the surfaces were coated with FG domain films. From the reproducibility of the compressive forces (considering the reproducibility of contact formation, Figure $3 \mathrm{C}$, and also the effect of thermal drifts during data acquisition), we estimate that the gap sizes thus determined are accurate to within $\pm 2 \mathrm{~nm}$.

Quantification of the Partitioning of Macromolecules. Having defined the polymer coating and the geometry of the confined space, we can now introduce the diffusing solute. Here, we have selected three probe molecules that have the same size but are expected to differ drastically in their interaction with FG domain films. GFP ${ }^{\mathrm{Std}}$ is the enhanced green fluorescent protein and is known to be weakly attracted to $\mathrm{FG}^{\mathrm{Nsp} 1}$ through a low level of nonspecific interactions. $\mathrm{GFP}^{\text {Inert }}$ is a mutant engineered to minimize such interactions. In contrast, $\mathrm{GFP}^{\mathrm{NTR}}$ is a mutant engineered to gain properties much like a nuclear transport receptor with an enhanced attraction to $\mathrm{FG}^{\mathrm{Nsp1}}$. These probe molecules originate from a recent study where the surface features of GFP were explored with respect to its NPC-translocation rate. ${ }^{37}$ Overall, a distinct correlation between NPC-passage rate and partitioning into macroscopic FG domain hydrogels was observed in these assays. In ref $37, \mathrm{GFP}^{\text {Inert }}$ is called SinGFP4A, and $\mathrm{GFP}^{\mathrm{NTR}}$ is called 7B3.

In a first instance, we focused on the distribution of GFP variants in the $\mathrm{FG}^{\mathrm{Nsp} 1}$ films. Figure $4 \mathrm{~A}$ and $\mathrm{B}$ show fluorescence micrographs of $\mathrm{GFP}^{\mathrm{Std}}$ surrounding the contact point between the planar and the hemispherical surface (center of image), for bare and $\mathrm{FG}^{\mathrm{Nsp} 1}$-functionalized surfaces, respectively. Although this is not immediately apparent in the micrographs, the corresponding radial intensity profiles clearly reveal that $\mathrm{GFP}^{\mathrm{Std}}$ was partly excluded from the FG domain film (Figure 4E). Equivalent micrographs of $\mathrm{GFP}^{\text {Inert }}$ and $\mathrm{GFP}^{\mathrm{NTR}}$ surrounding the contact point between FG domain functionalized surfaces are shown in Figure $4 \mathrm{C}$ and D, respectively. Similar to $\mathrm{GFP}^{\mathrm{Std}}$, $\mathrm{GFP}^{\text {Inert }}$ was also excluded from the FG domain film albeit to a greater extent. In contrast, as evident from the micrographs and the corresponding radial intensity profiles, GFP ${ }^{\mathrm{NTR}}$ was substantially enriched in the FG domain film. Supporting Figure S4 shows further controls for the specificity of the $\mathrm{FG}^{\mathrm{Nsp1}}$ film interactions with the used GFP variants.

For further analysis, we focused on the confined region in which the FG domain films that coat the planar and spherical surfaces overlap. Based on the geometry of the confined space (established by RICM for "soft" contact as shown in Figure 3F, and the additional $2 \times 2.3 \mathrm{~nm}=4.6 \mathrm{~nm}$ of the compacted $\mathrm{FG}^{\mathrm{Nsp1}}$ film in the contact area) and a thickness of $\sim 30 \mathrm{~nm}$ per uncompressed $\mathrm{FG}^{\mathrm{Nsp} 1}$ film (Supporting Figure S1) plus $2 \times 0.7$ $\mathrm{nm}=1.4 \mathrm{~nm}$ for the APTES functionalization, one can estimate that this zone extends $19 \mu \mathrm{m}$ from the center of the contact area.

Because the extension of the confocal volume in $z$ is much larger than the gap size, the intensities shown in Figure 4E can be expected to scale with the areal density of GFP molecules (i.e., GFP molecules per unit of projected area). Thus, by rescaling the intensity by the gap size, a measure of the GFP concentration within the gap volume can obtained. This data is shown in Figure 4F as a function of the gap size. In the case of $\mathrm{GFP}^{\mathrm{Std}}$ confined between bare surfaces the rescaled intensity is constant for gap sizes of $20 \mathrm{~nm}$ and more; it gradually decreases toward smaller distances and practically attains zero around $5 \mathrm{~nm}$. The observed trends are consistent with expectations for simple volume exclusion: GFP has a size of $5 \mathrm{~nm}$ and should thus not penetrate into gaps smaller than that, and depletion effects at the wall are expected to lead to a gradual increase in concentration for small gap sizes until a plateau corresponding to the bulk concentration is effectively reached. The match with these expectations lends support to the validity of the analytical approach. In addition, this control has the benefit of enabling conversion of the rescaled intensities into concentrations: by identifying the plateau value of $I_{\text {rescaled }}=3.5$ with the bulk concentration $c_{\text {bulk }}=2 \mu \mathrm{M}$, we have $c=2 \mu \mathrm{M} / 3.5 \times I_{\text {rescaled }}$.

In $\mathrm{FG}^{\mathrm{Nsp} 1}$ films, all GFP variants show a behavior that differs from $\mathrm{GFP}^{\mathrm{Std}}$ between bare surfaces: GFP ${ }^{\mathrm{Std}}$ and even more so $\mathrm{GFP}^{\text {inert }}$ are depleted, whereas GFP ${ }^{\mathrm{NTR}}$ is strongly enriched. Moreover, it is notable that the concentration of all GFP variants varies substantially with gap size. Figure $4 \mathrm{G}$ shows the same data as Figure $4 \mathrm{~F}$ but with the gap size converted to $\mathrm{FG}^{\mathrm{Nsp} 1}$ concentrations based on the known areal mass density of $320 \mathrm{ng} / \mathrm{cm}^{2}$ (corresponding to $5 \mathrm{pmol} / \mathrm{cm}^{2}$, or a root-meansquare distance between anchor points of approximately $6 \mathrm{~nm}$; Supporting Figure S1) for each of the two apposed $\mathrm{FG}^{\mathrm{Nsp} 1}$ 

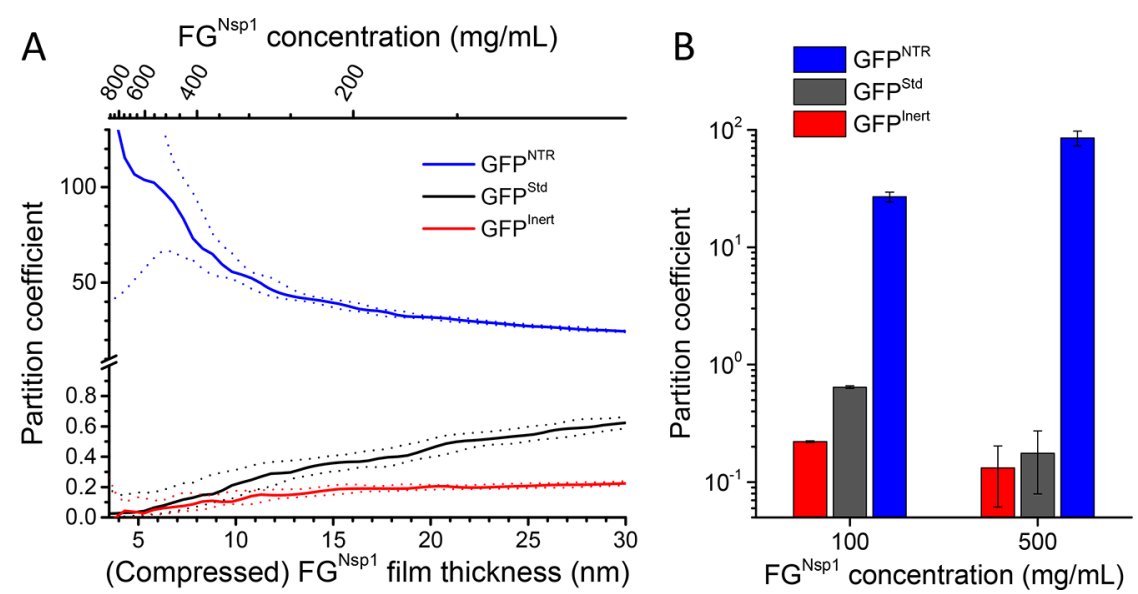

Figure 5. Analysis of GFP partitioning inside the polymer film with fluorescence microscopy. (A) Partition coefficients of GFP ${ }^{\text {Std }}$, GFP ${ }^{\text {NTR }}$, and $\mathrm{GFP}^{\mathrm{Inert}}$ inside the $\mathrm{FG}^{\mathrm{Nsp1}}$ film, calculated from the data in Figure $4 \mathrm{~F}$ as a function of the $\mathrm{FG}^{\mathrm{Nsp1}}$ concentration and (compressed) film thickness. (B) Comparison of partition coefficients for $\mathrm{FG}^{\mathrm{Nsp} 1}$ concentrations of approximately 100 and $500 \mathrm{mg} / \mathrm{mL}$, corresponding to a virtually uncompressed $\left(30 \mathrm{~nm}\right.$ thick) and strongly compressed $\left(6 \mathrm{~nm}\right.$ thick) $\mathrm{FG}^{\mathrm{Nsp1}}$ film, respectively. Mean values from two independent measurements per GFP variant are shown; error bars represent highest and lowest values obtained.

films. This plot represents the first main outcome of the PSCM method. A notable finding is that the concentration of $\mathrm{GFP}^{\mathrm{NTR}}$ increases with $\mathrm{FG}^{\mathrm{Nsp1}}$ concentration (with a linear dependence) over a substantial range of FG domain concentrations (from $\sim 100$ for the uncompressed film to $\sim 500 \mathrm{mg} / \mathrm{mL}$ ) before it shows the decrease that can be consistently seen for $\mathrm{GFP}^{\text {inert }}$ and $\mathrm{GFP}^{\mathrm{Std}}$. We note here that the end of the concentration scale in Figure $4 \mathrm{G}(1.2 \mathrm{mg} / \mathrm{mL})$ is already very close to a solvent-free polypeptide "melt" (density $1.4 \mathrm{mg} /$ $\mathrm{mL})$.

The partition coefficients (Figure 5), describing the partitioning of probe molecules between bulk solution and the FG domain 1 film, were determined from the data presented in Figure $4 \mathrm{~F}$ by calculating the ratios of intensities for $\mathrm{GFP}^{\mathrm{Std} / \mathrm{NTR} / \text { Inert }}$ in $\mathrm{FG}^{\mathrm{Nsp} 1}$ films and $\mathrm{GFP}^{\text {Std }}$ between bare surfaces. Figure 5A shows how the partition coefficient varies within the overlapping FG domain films. It was evident that

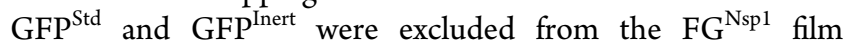
(partition coefficients $<1$ ) while $\mathrm{GFP}^{\mathrm{NTR}}$ was strongly enriched. Figure 5B illustrates how this differential effect is substantially enhanced when the FG domain film is compressed and thus more concentrated.

Quantification of Macromolecular Diffusion within Confined Polymer Layers. In contrast to conventional FRAP, line FRAP enables the analysis of spatial variations (i.e., along the bleached line) in diffusion in a single measurement with a resolution down to a few micrometers. We chose this approach as it is particularly well suited to probe how the diffusion varies with the gap size and, thus, the polymer film thickness and concentration.

The kymograph in Figure 6A shows a line FRAP data set for $\mathrm{GFP}^{\mathrm{NTR}}$ in $\mathrm{FG}^{\mathrm{Nsp} 1}$ films, where the imaged line was set to go through the center of the plane-sphere interface. The photobleached part of the line (cutting asymmetrically across the center) and the subsequent fluorescence recovery are readily visible in this crude presentation and demonstrate that $\mathrm{GFP}^{\mathrm{NTR}}$ is mobile everywhere in the confined area except in the $\sim 10 \mu \mathrm{m}$ wide central exclusion zone, which is hardly penetrated. Figure $6 \mathrm{~B}$ shows a recovery curve obtained by averaging over a $3 \mu \mathrm{m}$ wide section of the line (encased in white in Figure 6A). The best fit with a diffusion model (red line) assuming a mobile fraction $k$ with diffusion coefficient $D$ reproduces the data well and confirms that the vast majority of $\mathrm{GFP}^{\mathrm{NTR}}$ is mobile $(k=0.87 \pm 0.01$; note that equilibrium is not reached within the measured recovery phase of $1.2 \mathrm{~s}$ ). A possible explanation for the small fraction of apparently immobile $\mathrm{GFP}^{\mathrm{NTR}}(1-k=0.13 \pm 0.01)$ may be residual nonspecific interactions of the protein with the surfaces (Supporting Figure S4).

Performing such analyses along the bleached line reveals how the diffusion constant varies with the distance from the center and, thus, with the gap size or polymer concentration. Figure $6 \mathrm{C}$ illustrates how the diffusion coefficient of $\mathrm{GFP}^{\mathrm{NTR}}$ varies with the distance from the center based on Figure 6A. Figure $6 \mathrm{D}$ shows how the $\mathrm{GFP}^{\mathrm{NTR}}$ diffusion coefficient (averaged from multiple measurements) varies with FG domain film thickness and concentration. Reassuringly, the mobile fraction was consistently high across the full FG domain thickness range (and all measurements) at $k=0.84 \pm$ 0.02 (inset in Figure 6D), suggesting that possible surface effects do not skew the diffusion data appreciably.

In Figure 6D,E, it can be seen that GFP ${ }^{\mathrm{NTR}}$ diffuses with $D=$ $1.6 \pm 0.2 \mu \mathrm{m}^{2} / \mathrm{s}$ at the point where the FG domain films just overlap (film thickness $\approx 30 \mathrm{~nm} ; \mathrm{FG}^{\mathrm{Nsp} 1}$ concentration $\approx 100$ $\mathrm{mg} / \mathrm{mL}$ ) and that the diffusion constant decreased only moderately with increasing film compression and concentration. From analogous measurements with GFP $\mathrm{Gtd}^{\mathrm{Std}}$ (Supporting Figure S5) we estimate $D=6.5 \pm 1.9 \mu \mathrm{m}^{2} / \mathrm{s}$ for the unperturbed FG domain film. For comparison, the diffusion coefficient of GFP in aqueous solution has been determined by fluorescence correlation spectroscopy to be $D=90 \pm 3 \mu \mathrm{m}^{2}$ / s. ${ }^{38}$ Thus, the $\mathrm{FG}^{\mathrm{Nsp} 1}$ film reduces the diffusion of $\mathrm{GFP}^{\mathrm{Std}}$ (and likely also $\mathrm{GFP}^{\text {Inert }}$ ) by about an order of magnitude, while $\mathrm{GFP}^{\mathrm{NTR}}$ experiences a further reduction by a moderate few fold.

Salient Performance Features of PSCM. We have demonstrated that PSCM provides a radial gap profile (by RICM, with an accuracy in the gap size of a few nm; Figure 3), a radial solute concentration profile (by fluorescence microscopy; Figure 4E), and a radial solute diffusion profile (by line FRAP; Figure 6C). These data can be correlated for each radial position, and thus PSCM enables quantitation of a 

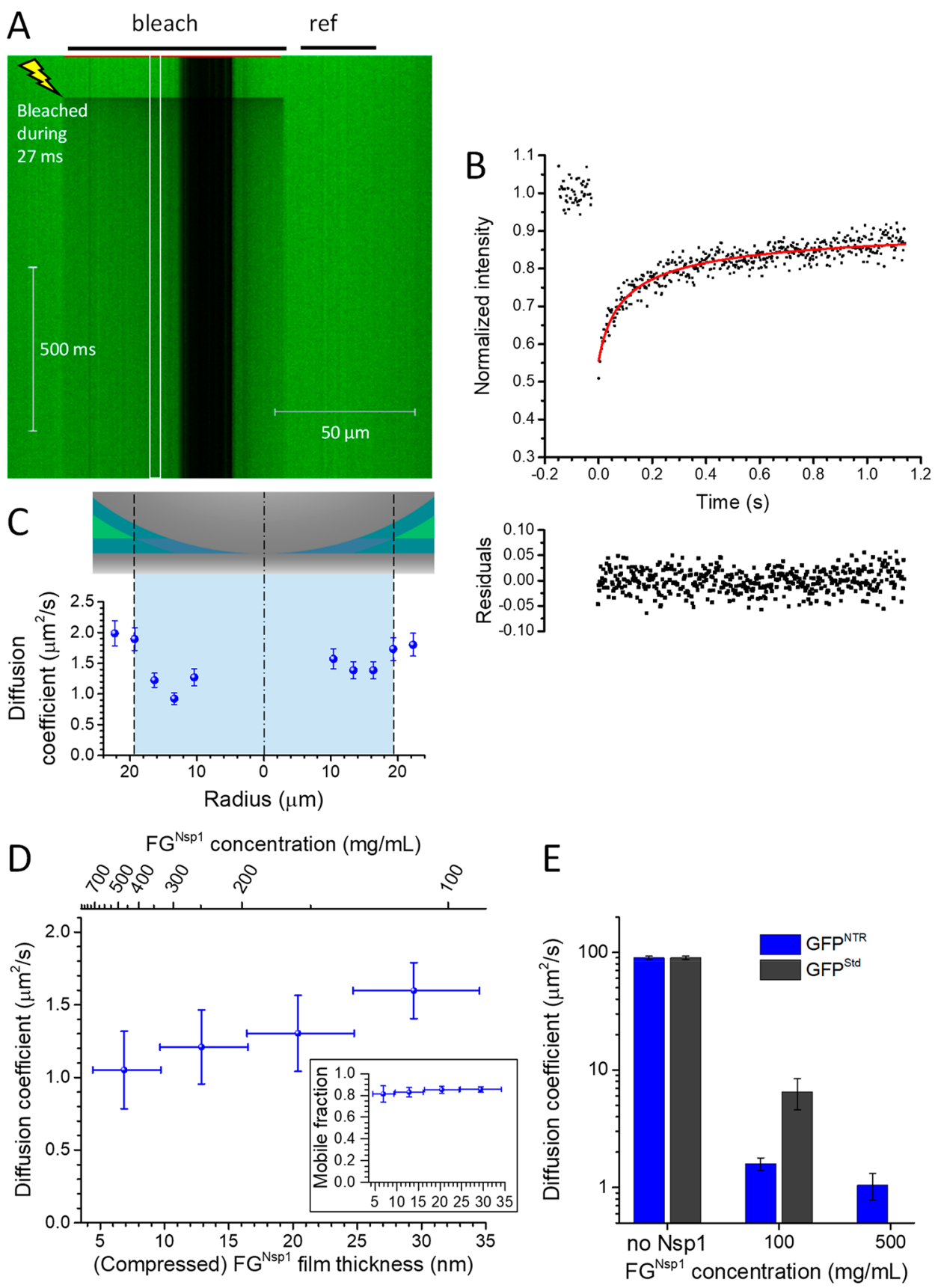

Figure 6. Analysis of macromolecular diffusion along confined polymer films by line FRAP. (A-D) Representative data for GFP ${ }^{\mathrm{NTR}}$ in an FG $^{\text {Nsp1 }}$ film to illustrate the data acquisition and analysis. (A) Kymograph of a scan line across the center of the plane-sphere contact area (cf. Figure 4D). The two lines on top mark parts of the scan line that are photobleached ("bleach"; yellow flash marks time point of bleaching) and used as reference to correct for bleaching during imaging ("ref"), respectively. (B) Fluorescence recovery curve (black dots) obtained from the data encased with a white box in (A). The best fit with the line FRAP model (red line; residuals from the fit are shown below) gives $D=1.4 \pm 0.2 \mu \mathrm{m}^{2} / \mathrm{s}, k=0.87 \pm 0.01$, and $K_{0}=0.88 \pm 0.03$. (C) GFP ${ }^{\mathrm{NTR}}$ diffusion coefficients at various distances from the contact point; error bars represent the standard error of the fit. (D) GFP ${ }^{\mathrm{NTR}}$ diffusion coefficient (main panel) and mobile fraction (inset) as a function of the $\mathrm{FG}^{\mathrm{Nsp} 1}$ concentration and (compressed) film thickness. Mean and standard deviations for 8 data points are shown ( 2 data points per image, left and right of the center, from a total of 4 images selected from 2 independent measurements); mobile fraction and bleaching parameter across these measurements were roughly constant: $k=0.84 \pm 0.02$ and $K_{0}=0.93 \pm 0.05$. (E) Comparison, for GFP ${ }^{\mathrm{NTR}}$ and GFP ${ }^{\text {std }}$, of diffusion constants in bulk solution ("no Nsp1"; taken from ref 38 ) and for FG $^{\text {Nsp1 }}$ concentrations of approximately 100 and $500 \mathrm{mg} / \mathrm{mL}$, corresponding to a virtually uncompressed (30 nm thick) and strongly compressed (6 nm thick) FG ${ }^{\mathrm{Nsp} 1}$ film, respectively.

wealth of information about the interaction of solutes with solvated polymer films in a single experiment. For polymer films of known thickness and/or surface coverage, the gap profile can be readily translated into a film compression profile and a polymer concentration profile, respectively. Partition coefficients can thus be obtained not only between the bulk and the uncompressed polymer phase but also as a function of the compression and concentration of the polymer phase (Figure 5). Importantly, in-plane diffusion becomes quantifiable with PSCM for the uncompressed polymer phase and as a 
function of the compression and concentration of the polymer phase (Figure 6D,E).

Several extensions to the presented capabilities of the PSCM method are conceivable. The determination of contact forces, and ultimately gap profiles, required the optical thickness of the fully compressed, solvent-free polymer interlayer to be determined with other methods. For our FG domain films, we used a combination of QCM-D and spectroscopic ellipsometry (see Supporting Figure S1 and Supporting Methods), though other techniques are also available. Alternatively one can quantify the optical thickness of the polymer interlayer using the RICM capability of PSCM, if the contact force is controlled by other means. A defined and constant contact force may be realized with some form of force balance, such as gravitation, for example. This enables the gap profile to be accurately determined for further analysis of solute diffusion and partitioning in less well characterized polymer interlayers (for details, see Supporting Methods, RICM analysis of a sphere pressing on a planar surface). Moreover, contact forces can be adjusted depending on the requirements of the polymer film of interest: for resilient films they can be made large enough such that essentially all solvent is being squeezed out in the contact area for a maximal range of compression to be probed; for fragile films forces can be kept small enough to avoid excessive damage.

In Figure 4G and Figure 5A we demonstrated that solute binding can be quantified as a function of polymer concentration in a single experiment. If such data are additionally acquired for a set of solute concentrations, then it becomes possible to obtain binding "isotherms" as a function of polymer concentration in a single experiment. While such data can also be obtained by other means, ${ }^{25}$ PSCM can provide them with higher throughput. Moreover, PSCM can probe in-film diffusion as a function of solute and polymer concentration. This not only enables the concentrationdependent diffusivity of the solute to be quantified but may also be exploited to measure how solutes affect the diffusivity of (fluorescently labeled) components of the polymer film itself.

For some applications, the possibility of determining the partition coefficient and/or diffusion constant of a molecule or nanoparticle within an uncompressed film may be particularly attractive. This requires a precise knowledge of film thickness to determine where exactly along the sphere-plane gap profile free solvent is excluded while polymers remain uncompressed. Our results (Figure 5A and Figure 6D) show that the measured values change gradually when transitioning from a compressed film (film thickness $<30 \mathrm{~nm}$ ) to an uncompressed film with some free solvent $(>30 \mathrm{~nm})$. Hence, an approximate knowledge of the film thickness is sufficient to obtain good estimates of the partition coefficient and diffusion constant within an uncompressed FG domain film. Other polymer films and solutes may though present very different partitioning and diffusion profiles; with a sharper transition it may become possible to infer the film thickness from the partitioning or diffusion profiles.

The presented confinement technique is versatile. It is compatible with most confocal and epi-fluorescence microscopy setups and can readily be added onto old or new microscopes. It is also compatible with all common methods to measure diffusion such as FRAP (including line FRAP), FCS (including raster image correlation spectroscopy, RICS $^{39}$ ), and SPT. For fluorescence-based SPT, conventional experiments usually require total internal reflection illumination (TIRF) to reduce background fluorescence signal from the bulk solution. ${ }^{40}$ With PSCM, a good signal-to-noise ratio can be expected even without TIRF because the confinement already effectively avoids background. This simplifies experiments, and for gap sizes smaller than $\sim 100 \mathrm{~nm}$ it is even more effective than TIRF. Also, with three-dimensional SPT, it would be possible to analyze in-plane diffusion as well as out-of-plane diffusion and hence to probe diffusion anisotropy in polymer films. Last but not least, the confinement technique should also be compatible with specialized nonfluorescent imaging and particle tracking modalities (e.g., photothermal microscopy ${ }^{41}$ ).

Insights into NTR-FG Domain Interactions and Functional Implications for NPC Permselectivity. In addition to establishing PSCM, this study also provided new insights into the dynamics of NTR-FG domain interactions.

The FG Domain Concentration Differentially Affects Uptake of NTRs and Inert Macromolecules. NTR binding depends on FG domain concentration in a nonmonotonic way. For the model NTR used here $\left(\mathrm{GFP}^{\mathrm{NTR}}\right)$, maximal binding occurred around $500 \mathrm{mg} / \mathrm{mL}$ (Figure 4G), a concentration that likely exceeds the FG domain concentration in the nuclear pore. In previous work, we had already found circumstantial evidence for such a nonmonotonic dependence for the NTR NTF2 in films made of an artificial, regular repeat of FSFG motifs. ${ }^{25}$ Collectively these data suggest that a nonmonotonic dependence is a common phenomenon, although further experiments will be required to quantify how this depends on NTR and FG domain types. We had previously shown that NTR binding to FG domain assemblies is determined by a balance of attractive interactions of NTRs with FG motifs and excluded volume repulsion. ${ }^{25}$ While both types of interaction can be expected to increase with FG domain density, our data suggest that the increase in attractive interactions dominates at low and intermediate FG domain concentrations (up to several $100 \mathrm{mg} / \mathrm{mL}$ ), whereas excluded volume repulsion takes over at the highest FG domain concentrations, thus giving rise to a nontrivial concentration dependence. For inert macromolecules, on the other hand, attractive interactions are minimal and uptake should decrease monotonously with FG domain concentration. This is indeed clearly evident for $\mathrm{GFP}^{\text {Std }}$ and GFP $^{\text {Inert }}$ (Figure 5A).

The opposite effect of FG domain concentration on the uptake of NTRs and inert macromolecules is intriguing, as it implies that there exists an optimal FG domain concentration where the NTR uptake is the most selective. In our specific experimental case we can define selectivity of uptake as the ratio of partition coefficients and see that the selectivity of $\mathrm{GFP}^{\mathrm{NTR}}$ over $\mathrm{GFP}^{\text {inert }}$ is $27 / 0.22 \approx 120$ at $100 \mathrm{mg} / \mathrm{mL} \mathrm{FG}^{\mathrm{Nsp} 1}$ (i.e., for the uncompressed FG domain film of $30 \mathrm{~nm}$ thickness) and $85 / 0.13 \approx 650$ at $500 \mathrm{mg} / \mathrm{mL} \mathrm{FG}^{\mathrm{Nsp} 1}$ (when the FG domain film is compressed to $6 \mathrm{~nm}$; Figure $5 \mathrm{~B}$ ). We note here that even more dramatic selectivity values have been reported for microphases of other FG domains ${ }^{37}$ and for real NTRs with $\mathrm{FG}^{\mathrm{Nsp1}}$ films; ${ }^{3,25}$ this however may arise at least to some extent because either the FG domain (in the microphases) or NTR (in FG ${ }^{\mathrm{Nsp} 1}$ films) was different. FG domains are known to exhibit a certain level of cohesiveness, which promotes the formation and determines the properties of FG domain phases ${ }^{34,42-45}$ and is also essential for the formation of a functional permeability barrier. ${ }^{27,44}$ Phases of the most cohesive natural FG domains indeed exhibit a rather high FG domain concentration (several $100 \mathrm{mg} / \mathrm{mL}$ ) yet still retain a 
significant amount of solvent. ${ }^{34,43}$ We propose an enhanced selectivity of NTR uptake as a previously unrecognized benefit of FG domain cohesiveness. It should be noted that the level of cohesiveness has to be balanced not only to maximize selectivity of NTR uptake but also because excessive cohesiveness may induce phase separation at the nanoscale and thus an effective breakdown of the permselectivity barrier in the NPC, as reported previously. ${ }^{34}$

FG Domain Phases Slow down NTR Diffusion Only Moderately. $\mathrm{GFP}^{\mathrm{NTR}}$ diffusion in $\mathrm{FG}^{\mathrm{Nsp} 1}$ films depends only weakly on the $\mathrm{FG}^{\mathrm{Nsp} 1}$ concentration, and the diffusion rate is not much lower than that of GFP in the cytosol $(D=6.1 \pm 2.4$ $\mu \mathrm{m}^{2} / \mathrm{s}$ for the cytoplasm in E. coli $\left.{ }^{46}\right)$. This finding is consistent with a moderate reduction in diffusion inside the NPC (as compared to the cytoplasm) for an import complex made from the NTRs importin $\alpha$ and $\beta$ and a GFP dimer model cargo. ${ }^{29}$ Future tests with other NTRs and FG domains can show if this is generally true. If so, this would reflect a distinctive adaption of NTR-FG domain interactions to the function of NTRs: enrichment in the nuclear pore, which is beneficial to transport but requires strong interactions with FG domains, is accomplished without a significant penalty on diffusion (which is generally slowed down by the attractive interactions). Most likely this is a consequence of the interactions of NTRs with individual FG motifs being very fast. ${ }^{31}$ With PSCM and designer FG domains and NTRs it now becomes possible to probe experimentally how NTR diffusion is defined by the multivalent nature of the interaction between NTRs and FG domains.

To estimate the magnitude of the effects that diffusion and partitioning in the FG domain phase have on fluxes $J$ across the NPC, we consider the simple theoretical model of the steadystate flux by Frey and Görlich, ${ }^{42}$ who arrived at $J=A D k_{\text {entry }} \Delta c /$ $\left(L k_{\text {exit }}+2 D\right)$, where $A$ and $L$ are the effective cross section and length of the NPC channel, respectively, $D$ is the diffusion constant inside the FG domain phase, $k_{\text {entry }}$ and $k_{\text {exit }}$ are the rate constants for entering and exiting the channel, and $\Delta c$ is the concentration difference across the channel. With the partition coefficient $P=k_{\text {entry }} / k_{\text {exit }}$, this equation can be recast into $J=A \Delta c /\left(L D^{-1} P^{-1}+2 k_{\text {entry }}^{-1}\right)$. If fluxes are limited by the diffusion through and exit from the pore $\left(k_{\text {entry }} \gg 2 D P / L\right)$, then $J \propto D P$. Under this condition, any moderate decrease in $D$ is overcompensated by a much larger increase in $P$, leading to an enhanced flux of NTRs compared to similar-sized inert molecules. Taking our results for GFP ${ }^{\mathrm{NTR}}$ and GFP inert $^{\text {at }} 100$ $\mathrm{mg} / \mathrm{mL} \mathrm{FG}^{\mathrm{Nsp} 1}$ as an example, we have a diffusion constant ratio of $1.6 \mu \mathrm{m}^{2} / \mathrm{s} /\left(6.5 \mu \mathrm{m}^{2} / \mathrm{s}\right) \approx 0.25$, a partition coefficient ratio of $27 / 0.22 \approx 120$, and thus a 30 -fold enhanced flux of $\mathrm{GFP}^{\mathrm{NTR}}$ over GFP inert . If instead entry into the pore is rate limiting $\left(k_{\text {entry }} \ll 2 D P / L\right)$, then $J$ does not depend on $D$ or $P$, and differences in flux instead arise from a larger entry rate of NTRs over inert molecules (not quantitated here).

The diffusion of $\mathrm{GFP}^{\mathrm{Std}}$ in the $\mathrm{FG}^{\mathrm{Nsp} 1}$ film is moderately reduced (by about an order of magnitude) compared to the bulk solution. This implies that the correlation length ("mesh size") within the $\mathrm{FG}^{\mathrm{Nsp} 1}$ film must be close to the size of GFP (cylinder with $4.2 \mathrm{~nm}$ length and $2.4 \mathrm{~nm}$ diameter). ${ }^{34}$ This is indeed quite reasonable considering the grafting density and volume density of the $\mathrm{FG}^{\mathrm{Nsp1}}$ film. It is also consistent with a moderate level of $\mathrm{GFP}^{\mathrm{Std}}$ and $\mathrm{GFP}^{\text {Inert }}$ exclusion from the $\mathrm{FG}^{\mathrm{Nsp1}}$ film (Figure 5B). For inert macromolecules that are significantly larger than the mesh size, polymer theory predicts the diffusion (and uptake) to be much reduced. ${ }^{47,48}$ PSCM now provides a tool to quantitate these effects and test the theoretical predictions for FG domain assemblies of defined composition and concentration.

\section{CONCLUSIONS}

In summary, we have presented an analytical method that allows quantitative characterization of macromolecular diffusion within (tens to hundreds of nanometers) thin solvated polymer coatings. The method can be integrated with conventional optical microscopes and is versatile. It provides quantitative information about the diffusion of macromolecules within the polymer coating and about the partitioning of macromolecules between the polymer film and the bulk solution. Thanks to the shape of the confined geometry, these parameters can also be mapped as a function of polymer film compression (and concentration) in a single experiment. The described methodology is generic and may find widespread use in the analysis of solvated polymer films and their interaction with fluorescent macromolecular probes. An obvious application in basic science is biomimetic model systems (e.g., for the nuclear pore permselectivity barrier, as presented here), where this method can provide insight into transport processes in complex polymeric environments. However, the potential use is much broader, and the methodology should find use in the development of functional coatings for a wide range of applications, from fundamental research in polymer and biological physics to everyday-life applications in biomaterials and paints.

Using the case of the nuclear pore permselectivity barrier we demonstrate direct quantitation of the diffusion coefficient of an NTR-like molecule within nanoscale assemblies of FG nucleoporins and demonstrate that the FG domain concentration sensitively affects the selectivity of NTR uptake. This data opens up avenues for further investigations to understand the physical mechanism underpinning the exquisite permselectivity of the nuclear pore complex.

\section{MATERIALS AND METHODS}

Materials. Chemicals were obtained from commercial sources and used without further purification. Ultrapure water (resistivity 18.2 $\mathrm{M} \Omega / \mathrm{cm}$ ) was used throughout. The FG domain of Nsp1 (amino acids 2 to 601$)$ from $S$. cerevisiae with a C-terminal $\mathrm{His}_{10}$ tag $\left(\mathrm{FG}^{\mathrm{Nsp1}}\right.$ $\mathrm{His}_{10} ; 64.1 \mathrm{kDa}$ ) was produced and purified as described earlier. ${ }^{3,34}$ For fluorescent labeling, the N-terminal cysteine of $\mathrm{FG}^{\mathrm{Nsp} 1}-\mathrm{His}_{10}$ was reacted with Atto 488 -maleimide as described previously. ${ }^{42} \mathrm{FG}^{\mathrm{Nsp1}}$ $\mathrm{His}_{10}$ variants were stored at concentrations between 11.5 and 15.6 $\mu \mathrm{M}(7.4$ and $10 \mathrm{mg} / \mathrm{mL})$ in $50 \mathrm{mM}$ Tris, $\mathrm{pH} 8$, supplementated with $6 \mathrm{M}$ guanidine hydrochloride $(\mathrm{GuHCl})$ at $-80^{\circ} \mathrm{C}$. Before use, the $\mathrm{FG}$ domains were diluted in working buffer $(10 \mathrm{mM}$ Hepes, $150 \mathrm{mM}$ $\mathrm{NaCl}, \mathrm{pH} 7.4)$ to a final concentration of $0.16 \mu \mathrm{M}(0.1 \mathrm{mg} / \mathrm{mL})$.

Three probe molecules derived from green fluorescent protein were used. GFP ${ }^{\text {Std }}$ is the well-known enhanced GFP. GFP ${ }^{\text {NTR }}$ and GFP ${ }^{\text {Inert }}$ are mutants that are described in detail in ref $37 . \mathrm{GFP}^{\mathrm{NTR}}$ (denoted 7B3 in ref 37) exhibits the qualities of an NTR in terms of facilitated transport through nuclear pores and in macroscopic FG domain hydrogels. GFP Inert (denoted SinGFP4A in ref 37) is "superinert" and is effectively excluded from nuclear pores and macroscopic FG domain hydrogels. Before use, the GFP samples were diluted in working buffer to a final concentration of $2 \mu \mathrm{M}$ unless otherwise stated.

Glass coverslips $\left(24 \times 24 \mathrm{~mm}^{2}\right.$, \#1.5, made from Schott D $263 \mathrm{M}$ glass) were purchased from Thermo Scientific. Rods of borosilicate glass (type 1 class A) with a diameter of $5 \mathrm{~mm}$ were purchased from VWR. These were cut into $25 \mathrm{~mm}$ long pieces, and ends polished to approximately hemispherical caps with a radius of curvature of 
approximately $3 \mathrm{~mm}$. The surfaces thus prepared were smooth on the nanometer scale, with a root-mean-square roughness of $0.4 \mathrm{~nm}$ as measured by atomic force microscopy (Supporting Figure S6A).

EDTA Functionalization of Glass Surfaces. Glass coverslips and glass rods with hemispherical caps to be functionalized with FG domains were prefunctionalized with EDTA, according to an established procedure, ${ }^{33}$ to allow binding of polyhistidine tagged proteins. Initially, the surfaces were cleaned by $10 \mathrm{~min}$ sonication in $2 \%$ SDS and water, respectively. After rinsing with water, surfaces were first blow dried using nitrogen gas $\left(\mathrm{N}_{2}\right)$ and then treated with UV/ozone (ProCleaner 220, BioForce Nanosciences, USA) for 30 min. A desiccator harboring $30 \mu \mathrm{L}$ of APTES (without any solvent) was purged with $\mathrm{N}_{2}$ gas for $2 \mathrm{~min}$. The glass surfaces were then placed inside, followed by purging with $\mathrm{N}_{2}$ for another $3 \mathrm{~min}$. The desiccator was sealed, and the surfaces were incubated for $1 \mathrm{~h}$. The surfaces were then sequentially incubated in four freshly prepared aqueous coupling solutions (0.5 M EDTA, $0.25 \mathrm{mM}$ EDC, $\mathrm{pH} 8.0$ ), once for $3.25 \mathrm{~h}$, twice for $2 \mathrm{~h}$, and then once for $15 \mathrm{~h}$. After the final incubation the surfaces were rinsed with water and blow dried with $\mathrm{N}_{2}$. This surface coating did not enhance the surface roughness appreciably (Supporting Figure S6B). The EDTA-functionalized surfaces were stored in air at room temperature until use.

Optical Microscopy and Setup of the Plane-Sphere Confinement Microscopy. All microscopy experiments were performed using an inverted laser scanning microscope (LSM 880; Zeiss, Oberkochen, Germany) equipped with a $40 \times$ oil immersion objective having a numerical aperture of 1.4 (Plan-Apochromat 40×/ 1.4 oil DIC M27). Images of $512 \times 512$ or $1024 \times 1024$ pixels were captured using a pixel dwell time of $2.06 \mu \mathrm{s}$. For fluorescence imaging the pinhole size was set to 5 airy units. This setting provided for robust alignment of the midplane of the confocal volume with the plane-sphere interface at a suitable lateral resolution $\left(r_{x y}=0.50 \pm\right.$ $0.04 \mu \mathrm{m}$ determined experimentally at $488 \mathrm{~nm}$ laser wavelength; Supporting Figure S7).

The sample chamber consisted of a custom-made PTFE holder to the planar bottom of which a suitably functionalized glass coverslip was attached using silicon glue (Twinsil; Picodent, Wipperfürth, Germany). The holder with coverslip was then mounted on the microscope stage. They formed the walls of a cylindrical cuvette of 10 $\mathrm{mm}$ diameter, the axis of which was coarsely aligned with the optical axis.

To form FG domain films, EDTA-functionalized planar and hemispherical surfaces were incubated first with $2 \mathrm{mM} \mathrm{NiCl}$ in working buffer $(15 \mathrm{~min})$ and then with $0.16 \mu \mathrm{M}(0.1 \mathrm{mg} / \mathrm{mL})$ $\mathrm{FG}^{\mathrm{Nsp1}}-\mathrm{His}_{10}$ in working buffer $(30 \mathrm{~min})$. After the latter incubation step, excess sample was removed by serial dilutions with working buffer. To visualize the FG domain film, $1 \mathrm{~mol} \%$ of fluorescently labeled $\mathrm{FG}^{\mathrm{Nsp1}}-\mathrm{His}_{10}$ was mixed into the $\mathrm{FG}^{\mathrm{Nsp1}}-\mathrm{His}_{10}$ solution in some experiments. Throughout the experiment, protein-coated surfaces were kept in working buffer to prevent drying. Probe molecules were added to reach a final concentration of $2 \mu \mathrm{M}$ unless otherwise stated.

One end of a suitably functionalized rod with hemispherical caps was lifted into the cylindrical cuvette with the aid of a micromanipulator (PatchStar; Scientifica, Uckfield, UK). Transmitted and reflected laser light served as guidance to facilitate coarse and fine alignment, respectively, of the rod axis with the optical axis before the spherical cap and the planar coverslip were brought into contact (Supporting Figure S2). Once contact between the surfaces was reached, the area of contact and its close surroundings (typically 150 $\times 150 \mu \mathrm{m}^{2}$ ) were imaged. In addition, RICM and FRAP experiments were carried out as described below.

The background fluorescence intensity was recorded with the focus position set $50 \mu \mathrm{m}$ below the solid-liquid interface of the planar surface, i.e., within the glass coverslip. The average fluorescence intensity of such images was determined using ImageJ software.

Reflection Interference Contrast Microscopy. The planesphere geometry allows for the use of RICM, a well-established technique ${ }^{49}$ that utilizes the interference pattern created by reflections at the apposed planar and curved interfaces to determine the gap profile between them. In conventional RICM applications, the typical size of the spherical probe is in the micrometer range, and RICM has previously been combined with colloidal probe atomic force microscopy, to study the mechanical properties of polymer brushes. ${ }^{50}$ In contrast, the hemispherical cap used in our setup has a radius in the millimeter range. Therefore, for RICM imaging the pinhole was opened to the maximum and the focus was positioned a few micrometers below the upper surface of the planar glass coverslip. This provided a high contrast image of the circular interference pattern (Newtonian rings) with minimal stray light. RIC micrographs with the interference patterns were analyzed with a custom-written algorithm implemented in LabView (described previously ${ }^{51}$ ) to quantify the effective height at the center of the plane-sphere interface. Data were fitted over an area of $170 \times 170 \mu \mathrm{m}^{2}$ typically encompassing six full interference fringe rings. Fixed input parameters for the algorithm were the radius of curvature of the hemispherical cap $(R=3 \mathrm{~mm})$, the wavelength $(\lambda=633 \mathrm{~nm})$, the pixel size, the refractive index of the buffer $(n=1.334)$, and the illumination numerical aperture (INA $=0.999$ ). The INA was experimentally determined by imaging the interference fringes formed between two nonparallel coverslips (air wedge in between the two surfaces) and by subsequently fitting the obtained intensity profile as described by Rädler et al. ${ }^{52}$ Adjustable parameters in the fitting routine were the effective RICM height along with two parameters accounting for background intensity, two parameters for amplitude normalization, and one parameter accounting for residual defocus and errors in $R$.

Fluorescence Recovery after Photobleaching in Line Mode. The diffusion of probe molecules within the overlapping polymer brushes was quantified using line FRAP. ${ }^{53}$ A single line, across the point of contact between the two surfaces, was imaged continuously. After a number of scans, a part of the line was bleached and the fluorescence recovery was then monitored.

Kymographs for line FRAP analysis were acquired as a times series of 512 line scans over 512 pixels. The first 50 line scans were used to acquire prebleach data. A selected part (260 pixels) of the line was then bleached using the maximal intensity of the $488 \mathrm{~nm}$ laser $(10$ bleach iterations; total bleach time of $27.2 \mathrm{~ms}$ ), and the remaining lines were used to monitor the fluorescence recovery. Fluorescence recovery profiles were extracted from the kymographs using ImageJ, and fits with the line FRAP equation were performed in Origin Pro (OriginLab, Northampton, MA, USA).

The normalized fluorescence intensity was determined by $I_{\text {norm }}(x$, $t)=\left(I_{\text {meas }}(x, t)-I_{\mathrm{bg}}\right) /\left(I_{\text {pre }}(x)-I_{\mathrm{bg}}\right)$, where $I_{\text {meas }}(x, t)$ is the measured intensity at position $x$ and time $t$ after bleaching, $I_{\mathrm{bg}}$ is the mean background intensity (measured by focusing inside the glass coverslip), and $I_{\text {pre }}(x)$ is the mean prebleach intensity (averaged over the scans prior to bleaching). This was further corrected for residual bleaching in the recovery phase as $I(x, t)=I_{\text {norm }}(x, t) /$ $I_{\text {norm,ref }}(t)$, where $I_{\text {ref }}(t)$ is the fluorescence intensity in the reference part of the line that was exempt from the deliberate 10 bleach iterations.

The fluorescence recovery in line FRAP was described according to $^{53}$

$$
\begin{aligned}
I(x, t)= & k \sum_{j=0}^{\infty} \frac{\left(-K_{0}\right)^{j}}{j !} r_{0 \mathrm{e}}\left[j r_{0 \mathrm{c}}^{2}+\left(1+2 j \frac{t}{\tau_{\mathrm{r}}}\right) r_{0 \mathrm{e}}^{2}\right]^{-1 / 2} \\
& +(1-k) I(x, 0)
\end{aligned}
$$

where $K_{0}$ is the bleaching parameter, $r_{0 c}$ is the imaging resolution, and $r_{0 \mathrm{e}}$ is the width of the bleached line. Moreover, the diffusion constant $D$ is obtained from the characteristic recovery time $\tau_{\mathrm{r}}=r_{0 \mathrm{e}}^{2} / 4 D, k$ is the mobile fraction, and $I(x, 0)$ is the fluorescence intensity immediately after bleaching. In our experiments, we set the bleached fraction to be relatively small such that $r_{0 \mathrm{e}} \approx r_{0 \mathrm{c}}$ (Supporting Figure S7), which simplifies the equation to 


$$
I(x, t)=k \sum_{j=0}^{\infty} \frac{\left(-K_{0}\right)^{j}}{j !}\left[1+j\left(1+2 \frac{t}{\tau_{\mathrm{r}}}\right)\right]^{-1 / 2}+(1-k) I(x, 0)
$$

Implicit to eq $2 \mathrm{a}$ is

$$
I(x, 0)=\sum_{j=0}^{\infty} \frac{\left(-K_{0}\right)^{j}}{j !}(1+j)^{-1 / 2}
$$

which ultimately gives

$$
\begin{aligned}
I(x, t)= & k \sum_{j=0}^{\infty} \frac{\left(-K_{0}\right)^{j}}{j !}\left[1+j\left(1+\frac{8 D}{r_{0 \mathrm{e}}^{2}} t\right)\right]^{-1 / 2} \\
& +(1-k) \sum_{j=0}^{\infty} \frac{\left(-K_{0}\right)^{j}}{j !(1+j)^{1 / 2}}
\end{aligned}
$$

The underpinning assumptions of this model have been discussed in detail in the original work. ${ }^{53}$ Of note here is that the bleaching efficiency can be expected to be homogeneous throughout the entire sample along the optical axis because the gap size between the apposed surfaces is generally much smaller than the confocal depth in the relevant area close to their contact. Moreover, to meet the requirement of fluorescence molecules being uniformly distributed, we averaged over sections along the bleached line that were wider than the extension of the diffusion front $(D t)^{-1 / 2}$. Also, we aimed for keeping the bleaching phase sufficiently short to avoid any significant recovery during that phase.

We note in passing that the mobile fraction $k$ is here defined following the common convention as the fraction of recovered fluorescence in the limit of $t \rightarrow \infty$ relative to the total bleached fluorescence at $t=0$. This can be appreciated from eq $2 \mathrm{a}$, where the infinite sum term converges to 1 in the limit of $t \rightarrow \infty$, giving $\lim _{t \rightarrow \infty} I(t)=k+(1-k) I(t=0)$, and thus $k=[I(t \rightarrow \infty)-I(t=0)] /$ $[1-I(t=0)]$.

When fitting with eq 3 , we neglected all terms of $j \geq 6$. This sped up the analysis and had a negligible influence on the results. Moreover, we fixed $r_{0 \mathrm{c}}=r_{0 \mathrm{e}}=r_{x y}=0.50 \mu \mathrm{m}$ (see Supporting Figure S7). The three adjustable parameters thus were $D, k$, and $K_{0}$. Normalized $\chi^{2}$ values typically were around 5 and residuals scattered evenly around 0 , indicating a good fit.

\section{ASSOCIATED CONTENT}

\section{(s) Supporting Information}

The Supporting Information is available free of charge at https://pubs.acs.org/doi/10.1021/acsnano.0c02895.

Supporting Figures S1 to S7, supporting methods (RICM analysis of a hemisphere pressing on a planar surface; estimate of compressive forces between hemisphere and plane; FG domain film thickness under strong compression), and supporting references (PDF)

\section{AUTHOR INFORMATION}

\section{Corresponding Author}

Ralf P. Richter - School of Biomedical Sciences, Faculty of Biological Sciences, School of Physics and Astronomy, Faculty of Engineering and Physical Sciences, Astbury Centre of Structural Molecular Biology, and Bragg Centre for Materials Research, University of Leeds, Leeds LS2 9JT, United Kingdom; 이이.org/0000-0003-3071-2837; Email: r.richter@ leeds.ac.uk

\section{Authors}

Rickard Frost - School of Biomedical Sciences, Faculty of Biological Sciences, School of Physics and Astronomy, Faculty of Engineering and Physical Sciences, Astbury Centre of Structural
Molecular Biology, and Bragg Centre for Materials Research, University of Leeds, Leeds LS2 9JT, United Kingdom; (1) orcid.org/0000-0002-8342-193X

Delphine Débarre - University Grenoble Alpes, CNRS, LIPhy, 38000 Grenoble, France

Saikat Jana - School of Biomedical Sciences, Faculty of Biological Sciences, School of Physics and Astronomy, Faculty of Engineering and Physical Sciences, Astbury Centre of Structural Molecular Biology, and Bragg Centre for Materials Research, University of Leeds, Leeds LS2 9JT, United Kingdom

Fouzia Bano - School of Biomedical Sciences, Faculty of Biological Sciences, School of Physics and Astronomy, Faculty of Engineering and Physical Sciences, Astbury Centre of Structural Molecular Biology, and Bragg Centre for Materials Research, University of Leeds, Leeds LS2 9JT, United Kingdom; (1) orcid.org/0000-0003-0634-7091

Jürgen Schünemann - Department of Cellular Logistics, Max Planck Institute for Biophysical Chemistry, 37077 Göttingen, Germany

Dirk Görlich - Department of Cellular Logistics, Max Planck Institute for Biophysical Chemistry, 37077 Göttingen, Germany

Complete contact information is available at:

https://pubs.acs.org/10.1021/acsnano.0c02895

\section{Author Contributions}

R.F. and R.P.R. conceived the study. All authors contributed to the design of the experiments. R.F., D.D., and R.P.R. invented the PSCM technology. J.S. produced the proteins. R.F., S.J., F.B., and R.P.R. performed the experiments. All authors contributed to the analysis of the data. R.F., D.D., and R.P.R. wrote the manuscript, and all authors commented on the manuscript.

\section{Notes}

The authors declare the following competing financial interest(s): European patent application no. 19315169.3 relates to the PSCM technology; the application has been filed on 19/12/2019 with University of Leeds and the Conseil National de la Recherche Scientifique (CNRS, France) as applicants, and R.F., D.D., and R.P.R. as inventors.

\section{ACKNOWLEDGMENTS}

This work was supported by the European Research Council (Starting Grant \#306435 "JELLY” and Proof of Concept Grant \#840295 "DIFFUSION" to R.P.R.) and the United Kingdom Biotechnology and Biological Sciences Research Council (BB/ R000174/1 to R.P.R.). O. Borisov (University of Pau-CNRS, France), L. Bureau (University Grenoble Alpes-CNRS, France), and P. Torstensson (Chalmers University of Technology, Gothenburg, Sweden) are acknowledged for fruitful discussions on polymer and solid mechanics.

\section{REFERENCES}

(1) Zhao, B.; Brittain, W. J. Polymer Brushes: Surface-Immobilized Macromolecules. Prog. Polym. Sci. 2000, 25, 677-710.

(2) Chen, W. L.; Cordero, R.; Tran, H.; Ober, C. K. 50th Anniversary Perspective: Polymer Brushes: Novel Surfaces for Future Materials. Macromolecules 2017, 50, 4089-4113.

(3) Eisele, N. B.; Frey, S.; Piehler, J.; Görlich, D.; Richter, R. P. Ultrathin Nucleoporin FG Repeat Films and Their Interaction with Nuclear Transport Receptors. EMBO Rep. 2010, 11, 366-372.

(4) Raynor, J. E.; Petrie, T. A.; Garcia, A. J.; Collard, D. M. Controlling Cell Adhesion to Titanium: Functionalization of Poly 
Oligo(Ethylene Glycol)Methacrylate Brushes with Cell-Adhesive Peptides. Adv. Mater. 2007, 19, 1724-1728.

(5) Welch, M. E.; Doublet, T.; Bernard, C.; Malliaras, G. G.; Ober, C. K. A Glucose Sensor via Stable Immobilization of the GOx Enzyme on an Organic Transistor Using a Polymer Brush. J. Polym. Sci., Part A: Polym. Chem. 2015, 53, 372-377.

(6) Higaki, Y.; Kobayashi, M.; Murakami, D.; Takahara, A. AntiFouling Behavior of Polymer Brush Immobilized Surfaces. Polym. J. 2016, 48, 325-331.

(7) Krishnan, S.; Weinman, C. J.; Ober, C. K. Advances in Polymers for Anti-Biofouling Surfaces. J. Mater. Chem. 2008, 18, 3405-3413.

(8) Kobayashi, M.; Takahara, A. Tribological Properties of Hydrophilic Polymer Brushes Under Wet Conditions. Chem. Rec. 2010, 10, 208-216.

(9) Whiting, G. L.; Snaith, H. J.; Khodabakhsh, S.; Andreasen, J. W.; Breiby, D. W.; Nielsen, M. M.; Greenham, N. C.; Friend, R. H.; Huck, W. T. S. Enhancement of Charge-Transport Characteristics in Polymeric Films Using Polymer Brushes. Nano Lett. 2006, 6, 573578.

(10) Wang, S. Q.; Jing, B. X.; Zhu, Y. X. Molecule Motion at Polymer Brush Interfaces from Single-Molecule Experimental Perspectives. J. Polym. Sci., Part B: Polym. Phys. 2014, 52, 85-103.

(11) Drummond, C.; Ruths, M. In Encyclopedia of Nanotechnology; Bhushan, B., Ed.; Springer: Dordrecht, The Netherlands, 2015.

(12) Kristiansen, K.; Donaldson, S. H., Jr.; Berkson, Z. J.; Scott, J.; Su, R.; Banquy, X.; Lee, D. W.; de Aguiar, H. B.; McGraw, J. D.; Degen, G. D.; Israelachvili, J. N. Multimodal Miniature Surface Forces Apparatus $(\mu \mathrm{SFA})$ for Interfacial Science Measurements. Langmuir 2019, 35, 15500-15514.

(13) Mukhopadhyay, A.; Bae, S. C.; Zhao, J.; Granick, S. How Confined Lubricants Diffuse During Shear. Phys. Rev. Lett. 2004, 93, 236105.

(14) Bae, S. C.; Wong, J. S.; Kim, M.; Jiang, S.; Hong, L.; Granick, S. Using Light to Study Boundary Lubrication: Spectroscopic Study of Confined Fluids. Philos. Trans. R. Soc., A 2008, 366, 1443-1454.

(15) Wong, J. S. S.; Hong, L.; Bae, S. C.; Granick, S. Fluorescence Recovery After Photobleaching Measurements of Polymers in a Surface Forces Apparatus. J. Polym. Sci., Part B: Polym. Phys. 2010, 48, 2582-2588.

(16) Leslie, S. R.; Fields, A. P.; Cohen, A. E. Convex Lens-Induced Confinement for Imaging Single Molecules. Anal. Chem. 2010, 82, 6224-6229.

(17) Berard, D.; McFaul, C. M.; Leith, J. S.; Arsenault, A. K.; Michaud, F.; Leslie, S. R. Precision Platform for Convex Lens-Induced Confinement Microscopy. Rev. Sci. Instrum. 2013, 84, 103704.

(18) Morrin, G. T.; Kienle, D. F.; Schwartz, D. K. Standalone Interferometry-Based Calibration of Convex Lens-Induced Confinement Microscopy with Nanoscale Accuracy. Analyst 2019, 144, $2628-2634$

(19) Adams, A. L.; Fischer, G. C.; Munoz, P. C.; Vroman, L. Convex-Lens-on-Slide: A Simple System for the Study of Human Plasma and Blood in Narrow Sapaces. J. Biomed. Mater. Res. 1984, 18, 643-654.

(20) Wahl, K. J.; Chromik, R. R.; Lee, G. Y. Quantitative In Situ Measurement of Transfer Film Thickness by a Newton's Rings Method. Wear 2008, 264, 731-736.

(21) Contreras-Naranjo, J. C.; Ugaz, V. M. A Nanometre-Scale Resolution Interference-Based Probe of Interfacial Phenomena between Microscopic Objects and Surfaces. Nat. Commun. 2013, 4, 1919.

(22) Stewart, M. Molecular Mechanism of the Nuclear Protein Import Cycle. Nat. Rev. Mol. Cell Biol. 2007, 8, 195-208.

(23) Grossman, E.; Medalia, O.; Zwerger, M. In Annual Review of Biophysics; Rees, D. C., Ed.; 2012; Vol. 41, pp 557-584.

(24) Denning, D. P.; Patel, S. S.; Uversky, V.; Fink, A. L.; Rexach, M. Disorder in the Nuclear Pore Complex: The FG Repeat Regions of Nucleoporins Are Natively Unfolded. Proc. Natl. Acad. Sci. U. S. A. 2003, 100, 2450-2455.
(25) Zahn, R.; Osmanovic, D.; Ehret, S.; Callis, C. A.; Frey, S.; Stewart, M.; You, C.; Goerlich, D.; Hoogenboom, B. W.; Richter, R. P. A Physical Model Describing the Interaction of Nuclear Transport Receptors with FG Nucleoporin Domain Assemblies. eLife 2016, 5, No. e14119.

(26) Vovk, A.; Gu, C.; Opferman, M. G.; Kapinos, L. E.; Lim, R. Y. H.; Coalson, R. D.; Jasnow, D.; Zilman, A. Simple Biophysics Underpins Collective Conformations of the Intrinsically Disordered Proteins of the Nuclear Pore Complex. eLife 2016, 5, No. e10785.

(27) Hülsmann, B. B.; Labokha, A. A.; Görlich, D. The Permeability of Reconstituted Nuclear Pores Provides Direct Evidence for the Selective Phase Model. Cell 2012, 150, 738-751.

(28) Kubitscheck, U.; Grunwald, D.; Hoekstra, A.; Rohleder, D.; Kues, T.; Siebrasse, J. P.; Peters, R. Nuclear Transport of Single Molecules: Dwell Times at the Nuclear Pore Complex. J. Cell Biol. 2005, 168, 233-243.

(29) Yang, W.; Musser, S. M. Nuclear Import Time and Transport Efficiency Depend on Importin $\beta$ Concentration. J. Cell Biol. 2006, 174, 951-961.

(30) Tu, L. C.; Fu, G.; Zilman, A.; Musser, S. M. Large Cargo Transport by Nuclear Pores: Implications for the Spatial Organization of FG-Nucleoporins. EMBO J. 2013, 32, 3220-3230.

(31) Milles, S.; Mercadante, D.; Aramburu, I. V.; Jensen, M. R.; Banterle, N.; Koehler, C.; Tyagi, S.; Clarke, J.; Shammas, S. L.; Blackledge, M.; Grater, F.; Lemke, E. A. Plasticity of an Ultrafast Interaction between Nucleoporins and Nuclear Transport Receptors. Cell 2015, 163, 734-745.

(32) Eisele, N. B.; Andersson, F. I.; Frey, S.; Richter, R. P. Viscoelasticity of Thin Biomolecular Films: A Case Study on Nucleoporin Phenylalanine-Glycine Repeats Grafted to a HistidineTag Capturing QCM-D Sensor. Biomacromolecules 2012, 13, 23222332.

(33) Ananth, A.; Genua, M.; Aissaoui, N.; Diaz, L.; Eisele, N. B.; Frey, S.; Dekker, C.; Richter, R. P.; Gorlich, D. Reversible Immobilization of Proteins in Sensors and Solid-State Nanopores. Small 2018, 14, No. e1703357.

(34) Eisele, N. B.; Labokha, A. A.; Frey, S.; Görlich, D.; Richter, R. P. Cohesiveness Tunes Assembly and Morphology of FG Nucleoporin Domain Meshworks - Implications for Nuclear Pore Permeability. Biophys. J. 2013, 105, 1860-1870.

(35) Wagner, R. S.; Kapinos, L. E.; Marshall, N. J.; Stewart, M.; Lim, R. Y. H. Promiscuous Binding of Karyopherin $\beta 1$ Modulates FG Nucleoporin Barrier Function and Expedites NTF2 Transport Kinetics. Biophys. J. 2015, 108, 918-927.

(36) Kapinos, L. E.; Schoch, R. L.; Wagner, R. S.; Schleicher, K. D.; Lim, R. Y. Karyopherin-Centric Control of Nuclear Pores Based on Molecular Occupancy and Kinetic Analysis of Multivalent Binding with FG Nucleoporins. Biophys. J. 2014, 106, 1751-1762.

(37) Frey, S.; Rees, R.; Schünemann, J.; Ng, S. C.; Funfgeld, K.; Huyton, T.; Görlich, D. Surface Properties Determining Passage Rates of Proteins through Nuclear Pores. Cell 2018, 174, 202-217.

(38) Junghans, C.; Schmitt, F.-J.; Vukojević, V.; Friedrich, T. Diffusion Behavior of the Fluorescent Proteins eGFP and Dreiklang in Solvents of Different Viscosity Monitored by Fluorescence Correlation Spectroscopy. Optofluid. Microfluid. Nanofluid. 2016, 3, 27-34.

(39) Brown, C. M.; Dalal, R. B.; Hebert, B.; Digman, M. A.; Horwitz, A. R.; Gratton, E. Raster Image Correlation Spectroscopy (RICS) for Measuring Fast Protein Dynamics and Concentrations with a Commercial Laser Scanning Confocal Microscope. J. Microsc. 2008, $229,78-91$.

(40) Holzmeister, P.; Acuna, G. P.; Grohmann, D.; Tinnefeld, P. Breaking the Concentration Limit of Optical Single-Molecule Detection. Chem. Soc. Rev. 2014, 43, 1014-1028.

(41) Boyer, D.; Tamarat, P.; Maali, A.; Lounis, B.; Orrit, M. Photothermal Imaging of Nanometer-Sized Metal Particles Among Scatterers. Science 2002, 297, 1160-1163. 
(42) Frey, S.; Görlich, D. A Saturated FG-Repeat Hydrogel Can Reproduce the Permeability Properties of Nuclear Pore Complexes. Cell 2007, 130, 512-523.

(43) Schmidt, H. B.; Görlich, D. Nup98 FG Domains from Diverse Species Spontaneously Phase-Separate into Particles with Nuclear Pore-Like Permselectivity. eLife 2015, 4, No. e04251.

(44) Frey, S.; Richter, R. P.; Görlich, D. FG-Rich Repeats of Nuclear Pore Proteins Form a Three-Dimensional Meshwork with HydrogelLike Properties. Science 2006, 314, 815-817.

(45) Patel, S. S.; Belmont, B. J.; Sante, J. M.; Rexach, M. F. Natively Unfolded Nucleoporins Gate Protein Diffusion Across the Nuclear Pore Complex. Cell 2007, 129, 83-96.

(46) Konopka, M. C.; Shkel, I. A.; Cayley, S.; Record, M. T.; Weisshaar, J. C. Crowding and Confinement Effects on Protein Diffusion. J. Bacteriol. 2006, 188, 6115-6123.

(47) Cai, L. H.; Panyukov, S.; Rubinstein, M. Mobility of Nonsticky Nanoparticles in Polymer Liquids. Macromolecules 2011, 44, 78537863.

(48) Cai, L. H.; Panyukov, S.; Rubinstein, M. Hopping Diffusion of Nanoparticles in Polymer Matrices. Macromolecules 2015, 48, 847862.

(49) Limozin, L.; Sengupta, K. Quantitative Reflection Interference Contrast Microscopy (RICM) in Soft Matter and Cell Adhesion. ChemPhysChem 2009, 10, 2752-2768.

(50) Attili, S.; Richter, R. P. Combining Colloidal Probe Atomic Force and Reflection Interference Contrast Microscopy to Study the Compressive Mechanics of Hyaluronan Brushes. Langmuir 2012, 28, 3206-3216.

(51) Davies, H. S.; Baranova, N. S.; El Amri, N.; Coche-Guerente, L.; Verdier, C.; Bureau, L.; Richter, R. P.; Debarre, D. An Integrated Assay to Probe Endothelial Glycocalyx-Blood Cell Interactions under Flow in Mechanically and Biochemically Well-Defined Environments. Matrix Biol. 2019, 78-79, 47-59.

(52) Rädler, J.; Sackmann, E. Imaging Optical Thicknesses and Separation Distances of Phospholipid Vesicles at Solid Surfaces. J. Phys. II 1993, 3, 727-748.

(53) Braeckmans, K.; Remaut, K.; Vandenbroucke, R. E.; Lucas, B.; De Smedt, S. C.; Demeester, J. Line FRAP with the Confocal Laser Scanning Microscope for Diffusion Measurements in Small Regions of 3-D samples. Biophys. J. 2007, 92, 2172-2183. 